\title{
THE EVOLUTIONARY HISTORY OF GALACTIC BULGES: PHOTOMETRIC AND SPECTROSCOPIC STUDIES OF DISTANT SPHEROIDS IN THE GOODS FIELDS
}

\author{
Lauren A. MacArthur, ${ }^{1}$ Richard S. Ellis, ${ }^{1}$ Tommaso Treu, ${ }^{2}$ Vivian U, ${ }^{3}$ Kevin Bundy, ${ }^{4}$ and Sean Moran ${ }^{5}$ \\ Received 2007 October 31; accepted 2008 February 26
}

\begin{abstract}
We report on the first results of a new study aimed at understanding the diversity and evolutionary history of distant galactic bulges in the context of now well-established trends for pure spheroidal galaxies. To this end, bulges have been isolated for a sample of 137 spiral galaxies within the redshift range $0.1<z<1.2$ in the GOODS fields. Using proven photometric techniques, we determine the characteristic parameters (size, surface brightness, profile shape) of both the disk and bulge components in our sample. In agreement with earlier work that utilized aperture colors, distant bulges show a broader range of optical colors than would be the case for passively evolving populations. To quantify the amount of recent star formation necessary to explain this result, we used DEIMOS to secure stellar velocity dispersions for a sizeable fraction of our sample. This has enabled us to compare the fundamental plane of our distant bulges with that for spheroidal galaxies in a similar redshift range. Bulges of spiral galaxies with a bulge-to-total luminosity ratio $(B / T)>0.2$ show similar patterns of evolution to those seen for pure spheroidals such that the stellar populations of all spheroids with $M>10^{11} M_{\odot}$ are consistent with a single major burst of star formation at high redshift $\left(z_{f} \gtrsim 2\right)$, while bulges with $M<10^{11} M_{\odot}$ must have had more recent stellar mass growth $(\sim 10 \%$ in mass since $z \sim 1)$. Although further data spanning a wider range of redshift and mass are desirable, the similarity between the assembly histories of bulges and low-mass spheroidals seems difficult to reconcile with the picture whereby the majority of large bulges form primarily via secular processes within spiral galaxies.
\end{abstract}

Subject headings: cosmology: observations — galaxies: bulges — galaxies: evolution — galaxies: formation — galaxies: high-redshift

Online material: color figure, machine-readable tables

\section{INTRODUCTION}

The history of galactic bulges remains a key issue in studies of the origin of the Hubble sequence. Originally thought to form at high redshift through dissipationless collapse (Eggen et al. 1962), their continued growth, as predicted in hierarchical models (Baugh et al. 1998), is consistent with the diversity observed in their presentday stellar populations (Wyse et al. 1997). Local data alone, however, cannot distinguish between quite different hypotheses for bulge formation, including secular processes triggered by interactions and the evolution of bars (Kormendy \& Kennicutt 2004; Combes 2007).

The assembly history of bulges is also central to understanding the strong correlations observed between the nuclear black hole mass and bulge properties (Magorrian et al. 1998; Gebhardt et al. 2000). The different physical scales involved in these local scaling relations represent a major theoretical challenge (MiraldaEscudé \& Kollmeier 2005). As ambiguities remain even if fairly precise observations are available for local samples, an important route to understanding this puzzle lies with undertaking observations at intermediate redshift. This is especially challenging for measurement of black hole masses where the sphere of influence remains unresolved (see Woo et al. 2006). However, as an alternative approach, it may be more practical to attempt to measure the growth history of the bulges in which they reside.

\footnotetext{
1 Department of Astrophysics, California Institute of Technology, MS 105-24, Pasadena, CA 91125; lam@astro.caltech.edu, rse@astro.caltech.edu.

2 Sloan Fellow; Packard Fellow; Department of Physics, University of California, Santa Barbara, CA 93106-9530; tt@physics.ucsb.edu.

3 Institute for Astronomy, University of Hawaii, Honolulu, HI 96822; vivian@ ifa.hawaii.edu.

4 Reinhardt Fellow; Department of Astronomy and Astrophysics, University of Toronto, Toronto, ON M5S 3H4, Canada; bundy@astro.utoronto.ca.

5 Department of Physics and Astronomy, Johns Hopkins University, Baltimore, MD 21218; moran@pha.jhu.edu.
}

The arrival of deep multicolor imaging data from the Hubble Space Telescope (HST) provided the first glimpse of the photometric properties of bulges at intermediate redshift. In an early paper, Ellis et al. (2001, hereafter EAD01) examined aperture colors of bulges in 68 suitably oriented isolated spirals with $I_{\mathrm{AB}}<24$ in the northern and southern Hubble Deep Fields (HDFs). The authors found a remarkable diversity in bulge colors over the redshift range $0.3<z<1$ (using both spectroscopic and photometrically determined redshifts), with few as red as a passively evolving track that matches the integrated colors of luminous spheroidal galaxies observed in the HDFs. ${ }^{6}$ EAD01 concluded that bulges have suffered recent periodic episodes of rejuvenation consistent with $15 \%-30 \%$ growth in stellar mass since $z \simeq 1$. These conclusions were challenged by Koo et al. (2005, hereafter $\mathrm{K} 05)$, who located 52 luminous $\left(I_{\mathrm{AB}}<24\right)$ bulges in the shallower but wider field Groth Strip Survey for which a more elaborate photometric decomposition was undertaken. They found that $85 \%$ of their field sample had uniformly red colors at $z \simeq$ $0.8[\delta(U-B) \simeq \pm 0.03]$, as red as present-day and distant cluster E/S0s. Only a minority (8\%) showed blue rest-frame colors, most of which occurred in interacting or merging systems. Earlier works based on photometric studies of the evolution of large spheroids out to $z \sim 0.6$ (Schade et al. 1996) and $z \sim 1$ (Kauffmann et al. 1996) also found conflicting results, the former finding spheroid evolution consistent with a passively evolving stellar population, the latter requiring strong evolution in the spheroid population since $z \sim 1$. Clearly, studies beyond those based purely on photometry and colors are required to resolve these discrepancies.

\footnotetext{
${ }^{6}$ We adopt the term "spheroidal galaxy" to denote pure elliptical systems, reserving "spheroidal component" where necessary to refer to bulges within galaxies also harboring a disk.
} 
In the interim, much has been learned about the luminosity dependence of evolution in the field spheroidal galaxy population. Treu et al. (2002), van Dokkum \& Ellis (2003), Treu et al. (2005a), Treu et al. (2005b, hereafter T05), van der Wel et al. (2005), and di Serego Alighieri et al. (2005) have undertaken comprehensive fundamental plane (FP) analyses of several hundred field galaxies to $z \simeq 1$. Whereas the most luminous spheroidal galaxies studied support the long-held view of early collapse and subsequent passive evolution (e.g., Bower et al. 1992), a surprising amount of recent star formation (SF) is necessary to explain the scatter and FP offsets for lower luminosity galaxies. T05 find that as much as $20 \%-40 \%$ of the present dynamical mass in systems with $M<10^{11} M_{\odot}$ formed since $z \simeq 1.2$. For these systems, spectroscopic signatures of recent SF are visible and several show resolved blue cores, consistent with recently accreted gasrich dwarfs.

The present paper aims to clarify the relationship between bulges and spheroidals in the light of the above work. The first part is motivated by the arrival of superior resolution multicolor Advanced Camera for Surveys (ACS) imaging data in the Great Observatories Origins Deep Survey (GOODS) fields; this represents a significant improvement over the WFPC2 data used by EAD01 and K05. The GOODS ACS data set offers the potential of securing improved photometric parameters. In particular, EAD01 chose not to undertake bulge-to-disk $(B / D)$ decomposition, arguing by example with local data (de Jong 1996) that aperture colors were adequate. In the present paper we revisit this discussion using a larger sample with equivalently deep but higher spatial resolution imaging allowing for photometric decompositions into bulge and disk parameters.

The second component is concerned with securing dynamical estimates of the bulge stellar masses from resolved spectroscopy. At intermediate redshifts, resolved spectroscopy of high quality is now possible thanks to instruments such as the Deep Imaging and Multi-Object Spectrograph (DEIMOS; Faber et al. 2003) on the Keck II telescope. Following recent progress in interpreting the mass assembly history of field spheroidals (e.g., T05), massto-light ratios $(M / L)$ determined from FP analyses are much superior to interpretations based solely on optical colors. Our goal is to secure the mass assembly history of a representative sample of intermediate-redshift bulges and to compare trends found with the integrated properties of field spheroidals.

A plan of the paper follows. In $\S 2$ we discuss the selection criteria we adopt for both the photometric and spectroscopic sample of bulges in the GOODS fields. In $\oint 3$ we describe the photometric techniques used for bulge/disk decomposition, taking into account the effects of the ACS point-spread function (PSF), and derive rest-frame properties ( $k$-corrections). In $\S 4$ we compare our photometric results with earlier measures of the diversity and star formation history (SFH) of intermediate-redshift bulges. In $\S 5$ we describe the spectroscopic measurements undertaken with DEIMOS and their reduction to the central stellar velocity dispersions necessary to construct the FP. Key issues of disk contamination, systematic rotation, and relative aperture size are discussed. In $\S 6$ we compare the FP of bulges with the trends now well established for more massive E/S0 galaxies. We discuss the implications of both our photometric and spectroscopic results in the context of various formation hypotheses in $\S 7$. We summarize our overall findings in $\S 8$.

Throughout this paper, for all distance-dependent quantities we adopt a flat cosmological model with $\Omega_{M}=0.3, \Omega_{\Lambda}=0.7$, and $H_{0}=65 \mathrm{~km} \mathrm{~s}^{-1} \mathrm{Mpc}^{-1}$. We note that both diagnostics considered in this study, colors and evolution of mass-to-light ratios, are independent of the Hubble constant. All magnitudes are in the AB system (Oke 1974) unless otherwise noted.

\section{DATA}

The primary data set for this study is the GOODS public v1.0 data release (Giavalisco et al. 2004), which provides deep imaging in four ACS passbands: F435W (B), F606W $(V)$, F775W $(i)$, and F850LP $(z)$. The depth and high resolution $\left(0.03^{\prime \prime} \mathrm{pixel}^{-1}\right.$ sampling) of the GOODS ACS photometry allow for an examination of the structure and luminosity parameters of spheroidal galaxies, and, in particular, spiral galaxy bulges can be isolated from their surrounding disk. Spectroscopic data are essential both for the photometric analysis, where redshifts are required to create rest-frame properties, and for the FP analysis, where precise bulge stellar velocity dispersions are required.

Spectroscopic data are drawn from two independent campaigns. The first is that of T05, who secured high signal-to-noise ratio (S/N) spectra of a magnitude-limited sample of field spheroiddominated galaxies in the northern GOODS field with $z_{\mathrm{AB}}<22.5$ spanning the redshift range $0.1<z<1.2$ (see Figs. 1 and 2 of T05 for a mosaic of this sample). Morphological classification was via visual inspection of the $z$-band images (see T05 for details). The integer-valued "T-type" system was adopted where $\mathrm{T}=\{0 \equiv \mathrm{E}, 1 \equiv \mathrm{E} / \mathrm{S} 0,2 \equiv \mathrm{S} 0,3 \equiv \mathrm{Sa}+\mathrm{b}, 4 \equiv \mathrm{S}, 5 \equiv \mathrm{Sc}+\mathrm{d}$, $6 \equiv \operatorname{Irr}\}$. Precision central stellar velocity dispersions were secured for 181 of these galaxies via one to two night exposures undertaken with DEIMOS during 2003 April 1-5 at the Keck observatory.

The second component of our bulge sample arises from later Keck campaigns during 2004 and 2005 dedicated to increasing the spiral sample for this project. Here we selected field spirals from the northern and southern GOODS fields according to visual classifications presented by Bundy et al. (2005). This sample was also limited at $z_{\mathrm{AB}}<22.5$ with the additional criterion of a known spectroscopic redshift from the Keck Team Redshift Survey (Wirth et al. 2004), whose selection was based purely on a magnitude limit $\left(R_{\mathrm{AB}}<24.4\right)$. This yielded a further target sample of 45 spirals within $0.1<z<0.7$. A mosaic of three-color ACS images for this second subset is shown in Figure 1.

The two spectroscopic subsets are naturally somewhat different. The first, from T05, is skewed to spheroid-dominated systems considered to be E/S0s in the originally released v0.5 images. Regardless of the original T-type classification (see T05 for details), all galaxies in the combined sample were independently visually examined as part of this analysis, in order to determine whether they were better described by one- (pure spheroidal) or two-component (spheroid plus disk) systems. Occasionally, particularly at high redshift, the visual inspection was not definitive. In such cases, the shape of the light profile was used as an additional guide by comparing one-component Sérsic fits to $B / D$ decompositions (see $\S 3.1$ ). The different fits and their residuals were compared to single- versus two-component fits of the bona fide spirals. Since we only consider the radial component of the light profiles, the 25 high-inclination $\left(i \gtrsim 70^{\circ}\right)$ spirals in both samples ( 17 from T05 and 8 from the new sample) are excluded from further analysis. Furthermore, a kinematic decomposition into separate components for the observed central velocity dispersion is not feasible. We thus restrict ourselves to galaxies with bulge-to-total ratio $(B / T)$ greater than 0.2 for the FP analysis to ensure that the effects of disk contamination are minimal (see $\S 5.3)$. This excludes a further 21 galaxies from the recent campaign and 25 from T05.

In summary, therefore, the photometric sample for this study comprises 193 galaxies in the redshift range $0.1<z<1.2$, of which 56 were modeled as single-component Sérsic profiles and 137 were decomposed into two components: exponential disk plus Sérsic bulge. The spectroscopic sample, a subset of 

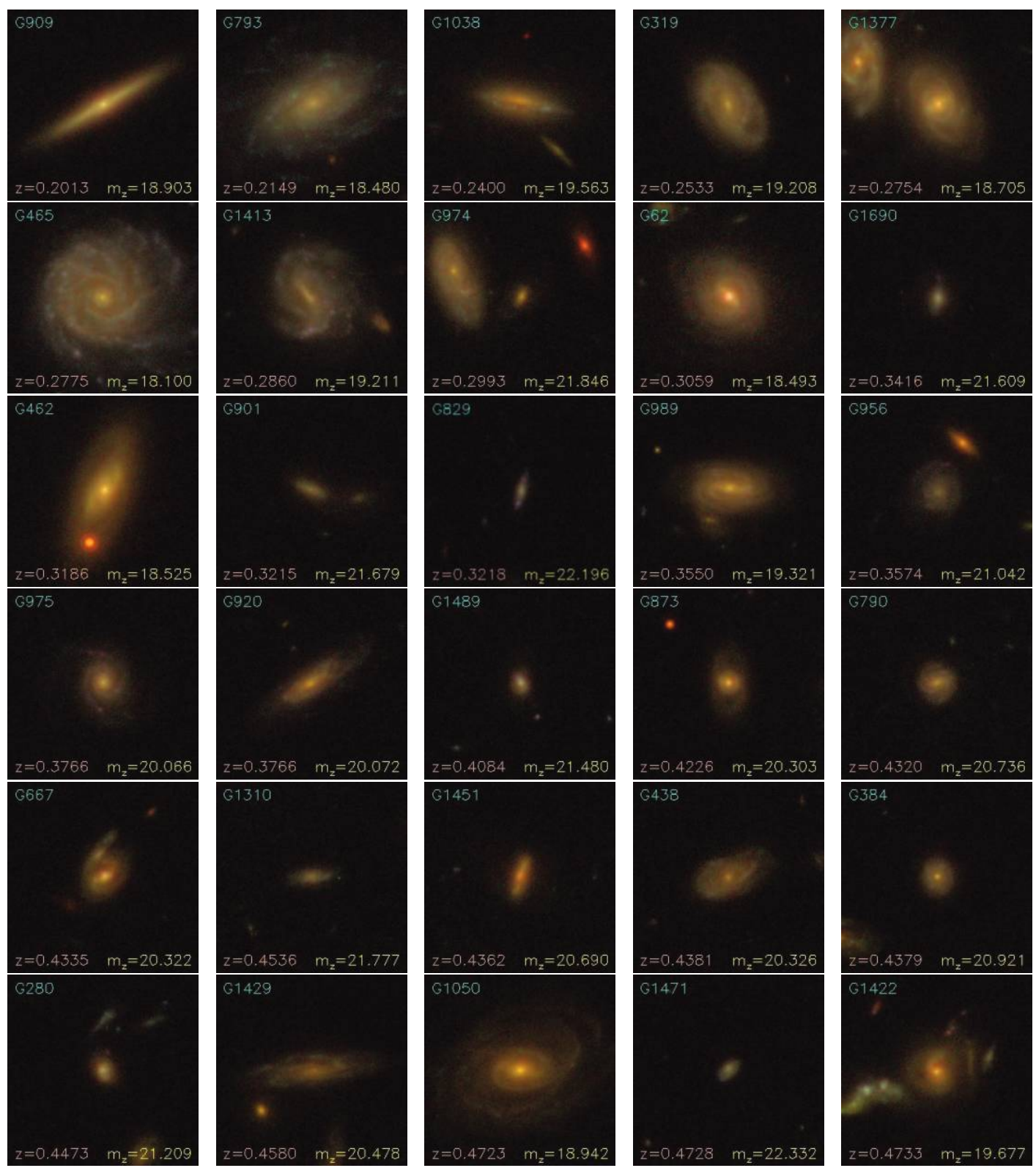

FIG. 1.- Mosaic of three-color composite ACS images $(\mathrm{R}=z, \mathrm{G}=i, \mathrm{~B}=V), 7^{\prime \prime}$ on a side, of the new GOODS bulge sample, sorted by redshift (lowest at top left, highest at bottom right). For each galaxy the internal ID (upper left corner), redshift (lower left corner), and total apparent $z$-band (F850LP) magnitude (lower right corner) are indicated.

the photometric sample from which the FP will be constructed, comprises 147 galaxies, 56 pure (single component) spheroidals and the spheroidal component of 91 two-component galaxies. Eight bulges are common to both spectroscopic subsamples, thereby offering a check on systematic errors (see $\S 5.2$ ). Figure 2 shows a histogram of the redshift distributions for both the photometric and spectroscopic samples. Finally, Table 1 lists positions, redshifts, T type, number of fit components, $B / T$, and total observed magnitudes (see $\S 3.3$ ) for all of our sample galaxies.

\section{PHOTOMETRIC ANALYSIS}

We begin with an examination of the deep ACS images in the GOODS v1.0 data release to derive the structural and luminosity parameters of the spheroidal components (defined here as the entire galaxy for single-component galaxies, or the isolated bulge for the two-component galaxies) of the full photometric sample. A key goal is the comparison of the photometric properties of our bulges with the spheroidals analyzed by $\mathrm{T} 05$.
Azimuthally averaged surface brightness (SB) profiles are extracted from the $z$-band images using the XVISTA package. ${ }^{7}$ Galaxy centers are determined interactively and fixed for the isophotal fit, but variable position angles (PAs) and ellipticities $(\epsilon=1-b / a$, where $a$ and $b$ are, respectively, the semimajor and semiminor axes) are permitted at each isophote. SB profiles are traced to $\sim 24-$ $26 \mathrm{mag} \operatorname{arcsec}^{-2}$, corresponding to a systematic SB error of $\lesssim 0.1 \mathrm{mag} \operatorname{arcsec}^{-2}$. Profiles were extracted in at least $z$ and $i$ for each galaxy in our sample, and most $z \lesssim 0.5$ also have adequate $V$ and $B$ profiles.

\subsection{Light Profile Modeling}

Surface brightness profiles are modeled using techniques described in detail in MacArthur et al. (2003, hereafter M03). A brief description is provided here.

\footnotetext{
${ }^{7}$ Developed at Lick Observatory. As of writing, XVISTA is maintained and distributed by Jon Holtzman at New Mexico State University and can be downloaded from http://ganymede.nmsu.edu/holtz/xvista/.
} 

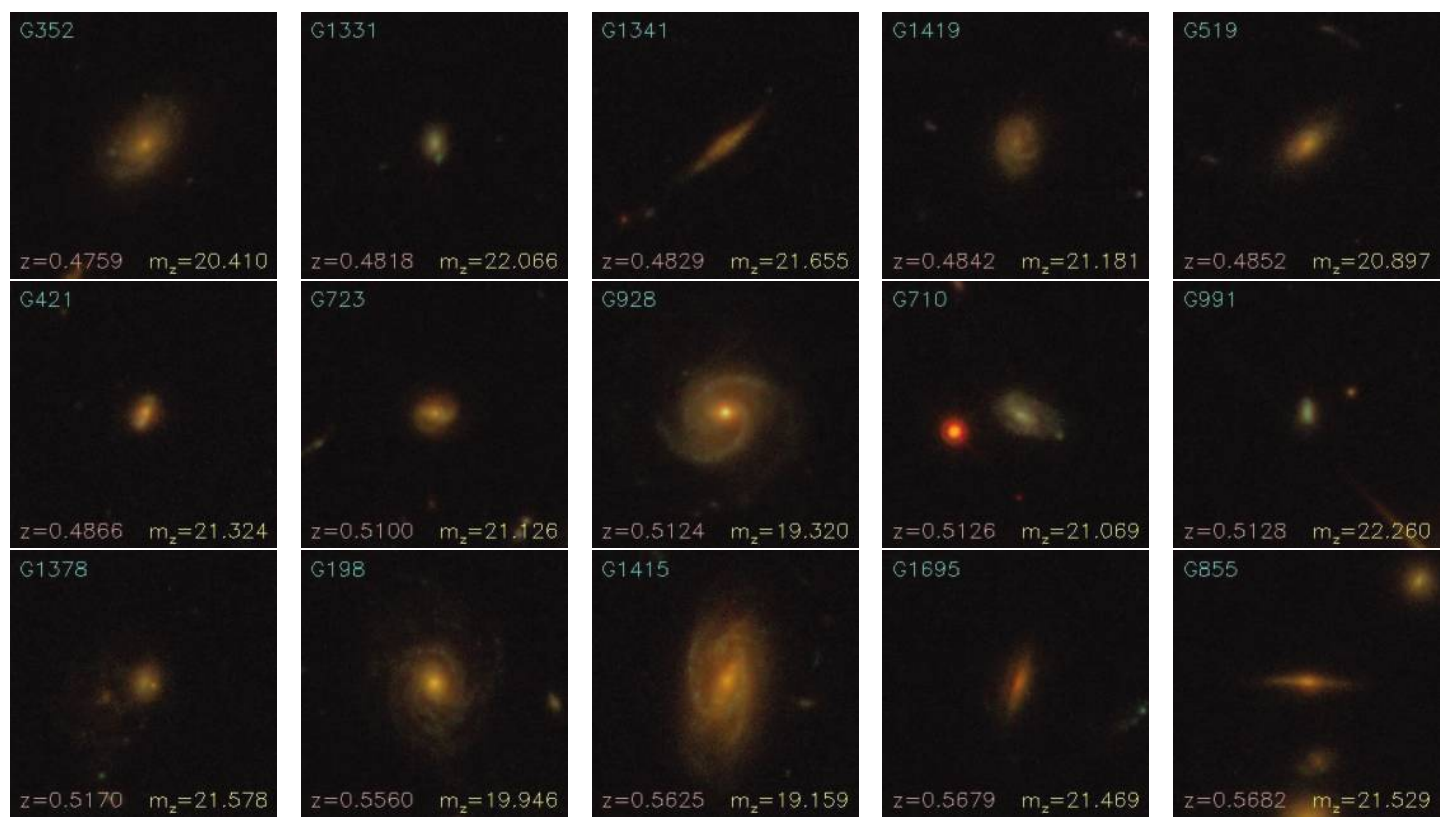

Fig. 1-Continued

Our profile modeling algorithm either reduces one-dimensional (1D) projected galaxy luminosity profiles into a single-component Sérsic profile or decomposes bulge and disk components simultaneously using a nonlinear Levenberg-Marquardt least-squares fit to the logarithmic intensities (i.e., magnitude units). Random SB errors are accounted for in the (data - model) minimization, whereas systematic errors such as uncertainties in the sky background and PSF measurements are accounted for separately by performing decompositions with the nominal values, as well as \pm their respective errors (see $\S 3.2 .2$ ).

A fundamental aspect of the profile decompositions is the choice of fitting functions. The exponential nature of disk profiles has been established observationally both locally (Freeman 1970; Kormendy 1977; de Jong 1996; M03) and at high $z$ (Elmegreen

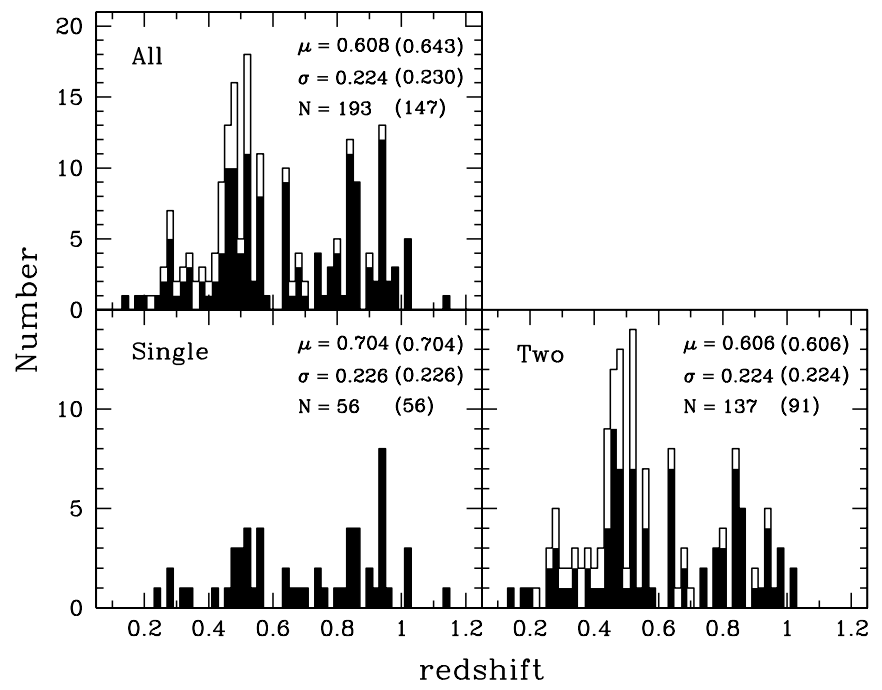

FIG. 2.- Histograms of sample redshift distribution. Top: full sample; bottom left: single-component galaxies; bottom right: two-component galaxies. Open histograms refer to the photometric sample; filled histograms and numbers in parentheses refer to the spectroscopic sample. et al. 2005). Hence, here we model the disk light, in magnitudes, as

$$
\mu_{d}(r)=\mu_{0}+2.5 \log _{10}(e)\left(\frac{r}{h}\right)
$$

where $\mu_{0}\left(\equiv-2.5 \log _{10} I_{0}\right)$ and $h$ are the disk central surface brightness (CSB) and scale length, respectively, and $r$ is the galactocentric radius measured along the major axis.

The appropriate form for spiral bulges and spheroidal galaxies is, however, less clear. Historically, all spheroids were modeled with the de Vaucouleurs $r^{1 / 4}$ profile, which has proven to be a good description for the shape of luminous elliptical galaxies. However, higher resolution studies have revealed a range of profile shapes for both E/S0s and spiral bulges, which generally correlates with the luminosity (Caon et al. 1993; Andredakis et al. 1995; Courteau et al. 1996; Graham 2001; M03). Such a range is also supported by numerical simulations of two-component (halo plus stars) dissipationless collapse (Nipoti et al. 2006) when mergers and secular evolution of disk material are accounted for (Scannapieco \& Tissera 2003; Debattista et al. 2006).

To ensure the most general form for the bulge SB profile, we adopt the formulation of Sérsic (1969), which, in magnitudes, becomes

$$
\mu_{b}(r)=\mu_{e}+2.5 \log _{10}(e) b_{n}\left[\left(\frac{r}{r_{e}}\right)^{1 / n}-1\right],
$$

where $\mu_{e}\left(\equiv-2.5 \log _{10} I_{e}\right)$ is the SB at the effective radius, $r_{e}$, enclosing half the total extrapolated luminosity, ${ }^{8}$ and $b_{n}$ is chosen to ensure that

$$
\int_{0}^{\infty} I_{b}(r) 2 \pi r d r=2 \int_{0}^{r_{e}} I_{b}(r) 2 \pi r d r
$$

\footnotetext{
${ }^{8}$ For a pure exponential disk, $r_{e}=1.678 h$
} 
TABLE 1

Galaxy Sample Parameters

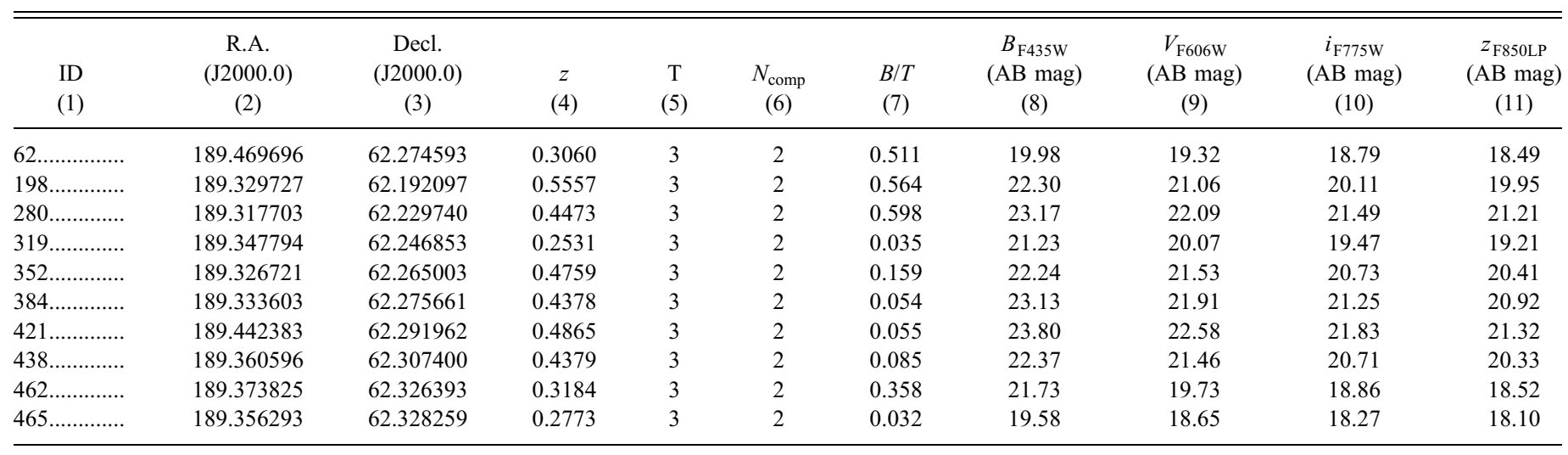

Notes.—Col. (1): Internal galaxy ID. Cols. (2) and (3): Right ascension and declination in J2000.0 epoch coordinates. Col. (4): Spectroscopic redshift. Col. (5): Galaxy $\mathrm{T}$ type according to T05. Col. (6): Number of components in decomposition $(1 \equiv$ single Sérsic profile in fit; $2 \equiv$ simultaneous Sérsic bulge plus exponential disk decomposition). Col. (7): Galaxy bulge-to-total ratio. Cols. (8)-(11): Total observed galaxy magnitudes corrected for Galactic extinction (see Table 2) in all four GOODSACS filters. Table 1 is published in its entirety in the electronic edition of the Astrophysical Journal. A portion is shown here for guidance regarding its form and content.

We use the approximation for $b_{n}$ given in Appendix A of M03, which is good to 1 part in $10^{4}$ for $n>0.36$ and 2 parts in $10^{3}$ for $n \leq 0.36$.

It has become customary to express the disk parameters in terms of scale length and CSB ( $h$ and $\mu_{0}$ ), while the spheroid is described in terms of effective parameters $\left(r_{e}\right.$ and $\left.\mu_{e}\right)$. We adopt this formalism, thus parameters with subscript " $e$ " refer to the bulge or spheroid. We use a capital $R_{e}$ to indicate radii expressed in physical units (usually kpc).

For the FP analysis in the following sections, we also need to compute the average $\mathrm{SB}$ within the effective radius. For the Sérsic profile this is computed as

$$
\mathrm{SBe}=-2.5 \log _{10}\left\langle I_{e}\right\rangle,
$$

where

$$
\left\langle I_{e}\right\rangle=\frac{I_{e} \exp \left(b_{n}\right) n \Gamma(2 n)}{b_{n}^{2 n}} .
$$

The best-fit parameters of the (data - model) comparison are then those that minimize the reduced $\chi^{2}$ merit function,

$$
\chi_{\nu}^{2}=\frac{1}{N-M} \sum_{i=1}^{N}\left[\frac{\mu_{\mathrm{gal}}\left(r_{i}\right)-\mu_{\mathrm{model}}\left(r_{i} ; h, \mu_{0}, r_{e}, \mu_{e}, n\right)}{\sigma_{i}}\right]^{2},
$$

where $N$ is the number of radial data points used, $M$ is the number of free parameters (i.e., $N-M=\nu \equiv$ degrees of freedom), and $\sigma_{i}$ is the statistical error at each SB level. From here on the $\nu$ subscript is omitted and the $\chi^{2}$ variable refers to a $\chi^{2}$ per degree of freedom.

\subsection{Fitting Procedure}

Constraining the five structural parameters of spiral galaxies is a challenge even at low redshift. At higher $z$, the lower (relative) resolution and depth limitations of the images render the challenge even greater. In order to avoid discrepant fits between different passbands, some workers have opted to perform fits simultaneously in all bands. The scale parameters are held fixed for the decompositions in all passbands, and only the flux levels are allowed to vary to obtain colors (e.g., K05). This procedure implies that there are no significant color gradients and that the bulge shapes are identical at all wavelengths. However, just as any morphological description of galaxies (e.g., Hubble types) depends on the wave band, intrinsic structural parameters have also been shown to vary with wavelength as a result of stellar population and dust extinction effects (e.g., MacArthur et al. 2004). Thus, multiwavelength information and independent fits at each wavelength are required for any accurate description of galactic structural parameters.

An additional narrowing of the parameter space is often accomplished by fixing the bulge shape to be that of a de Vaucouleurs profile (Sérsic $n=4$ ). While this may be justified for luminous spheroidal galaxies and earliest type spiral bulges, our sample contains a range in spheroidal galaxy luminosity and extends to late-type spirals that have been shown to have bulge profiles closer to that of an exponential (e.g., M03), thus such a constraint would be inappropriate. Physical differences in the shape and size of spheroids among galaxies are also expected depending on how they were formed. Formation by dissipationless collapse (Nipoti et al. 2006) or accretion processes (e.g., major/minor mergers) can account for steeply rising ( high- $n$ ) light profiles in the central parts of galaxies (e.g., Aguerri et al. 2001), while secular evolution of disk material (possibly triggered by a satellite) would yield shallower distributions ( $n \simeq 1-2$ ) of the central light (Scannapieco \& Tissera 2003; Eliche-Moral et al. 2006). The formation of small bulges is indeed largely attributed to secular processes and redistribution of disk material (see Kormendy \& Kennicutt 2004 and references therein).

An important constraint in analyzing our present data set is the need to compare the evolutionary characteristics of our new bulge sample with a similar sample of E/S0s (from T05). Ideally, the analysis techniques should be identical. In T05, structural homology was assumed and all profiles were modeled with a fixed $n=4$ profile, to conform with the standard practice for traditional studies of the FP of early-type galaxies in the local universe (e.g., Dressler et al. 1987; Djorgovski \& Davis 1987; Jørgensen et al. 1996, hereafter J96). However, the goal of this paper is to extend the study to bulges, which are typically best modeled as lower $n$ Sérsic profiles. Thus, to ensure homogeneity between the analysis of bulges and spheroidals, we have remodeled the light profiles of the T05 galaxies using the Sérsic profile for the spheroid component and adding a disk component when necessary. We find a range of best-fit Sérsic parameters for these early-type galaxies 
spanning $\sim 0.9<n<3.4$ (see Fig. $5 d$ below). The fact that the range in the Sérsic $n$ parameter found here does not extend as high as the de Vaucouleurs $n=4$ shape often assumed for elliptical galaxies deserves some comment. The recent literature on the shapes of elliptical galaxies reveals that the Sérsic $n$ for earlytype galaxies in fact peaks at $n \sim 2-3$ (e.g., Graham \& Guzmán 2003; Trujillo et al. 2004, hereafter T04; Ferrarese et al. 2006, hereafter F06). Because of the correlation between $n$ and total magnitude, the higher $n$ galaxies are also at the bright end of the luminosity function and tend to be the rarer cases most often found at the centers of massive clusters. Given that our sample is limited to field galaxies, we would not expect to find many high- $n$ systems; thus, the distribution found seems reasonable compared with the lower $z$ studies.

The following sections detail the fitting techniques, which are specific to our intermediate-redshift sample and are based exclusively on results from $1 \mathrm{D} B / D$ decompositions. The fact that we do not attempt to model nonaxisymmetric shapes (bars, rings, oval distortions) lessens the need for more computationally intensive two-dimensional (2D) $B / D$ decompositions (extensive simulations in M03 showed no improvements using the 2D over the 1D decomposition method when modeling axisymmetric structures). The measured parameters and correlations among them are discussed in $\S 3.5$.

\subsubsection{Initial Estimates}

In order to determine the range of best-fitted bulge and disk parameters, we need to assist the minimization program in finding the lowest possible (data - model) $\chi^{2}$ (see M03). We base our initial estimates for the disk parameters $h$ and $\mu_{0}$ on a "marking the disk" technique, where the linear portion of a luminosity profile is "marked" and the selected range is fitted using standard least-squares techniques to determine its slope. The baseline adopted for these fits is $0.25 r_{\max }-r_{\max }$. The inner boundary is chosen to exclude the major contribution of a putative bulge or a Freeman (1970) type II profile dip, and $r_{\max }$ is the radius at which the surface brightness error is greater than $0.1 \mathrm{mag} \operatorname{arcsec}^{-2}$.

Flexibility in the bulge initial parameters is, however, limited by PSF and sampling effects; thus, the Sérsic $n$ exponent cannot be fitted as a free parameter. We therefore hold $n$ fixed in the decompositions and explore the full range of $0.1<n<6.0$ in steps of $n=0.1$. A grid search is performed to select the best fit. As in M03, for each decomposition (at fixed $n$ ) we explore four different sets of initial bulge parameter estimates to protect against local minima in the parameter space.

\subsubsection{Point-Spread Function and Sky Treatment}

Bulges of late-type spirals are small and their luminosity profiles can be severely affected by the PSF. The PSF is accounted for by convolving the model light profiles with a radially symmetric Gaussian PSF prior to comparison with the observed profile.

The PSFs in the GOODS ACS images were estimated using a modified version of the Tiny Tim HST PSF modeling software ${ }^{9}$ (Rhodes et al. 2007). For each of the four GOODS filters, 2500 artificial stars were inserted across the ACS WFC field with a telescope focus value of $-2 \mu \mathrm{m}$ (i.e., a primary/secondary spacing $2 \mu \mathrm{m}$ smaller than nominal). SExtractor (Bertin \& Arnouts 1996) was then used to measure the FWHM of those PSFs. ${ }^{10}$

\footnotetext{
${ }^{9}$ See http://www.stsci.edu/software/tinytim/tinytim.html.

10 Note that this procedure does not account for the long-wavelength "halo" observed in the PSF of the reddest ACS bands (Sirianni et al. 1998).

As such, whenever we consider observed spheroid colors, we restrict ourselves to the minimally affected F606W $(V)$ and F775W $(i)$ filters.
}

TABLE 2

Adopted Quantities Specific to the Four GOOdS-ACS Filters

\begin{tabular}{|c|c|c|c|c|c|}
\hline \multirow[b]{2}{*}{ Filter } & \multicolumn{2}{|c|}{$A_{\lambda}^{\mathrm{a}}(\mathrm{mag})$} & \multicolumn{2}{|c|}{$\mathrm{PSF}^{\mathrm{b}}(\operatorname{arcsec})$} & \multirow{2}{*}{ 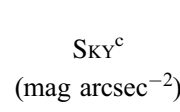 } \\
\hline & North & South & $\mu$ & $\sigma$ & \\
\hline F435W............. & 0.0531 & 0.0336 & 0.071 & 0.006 & 23.4 \\
\hline 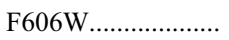 & 0.0354 & 0.0233 & 0.080 & 0.006 & 22.7 \\
\hline F775W & 0.0254 & 0.0165 & 0.088 & 0.003 & 22.2 \\
\hline F850LP & 0.0165 & 0.0114 & 0.093 & 0.003 & 22.2 \\
\hline
\end{tabular}

${ }^{\text {a }}$ Galactic foreground extinction using the reddening values, $A_{\lambda}$, of Schlegel et al. (1998) and interpolating to the effective wavelength of the GOODS-ACS filters.

b ACS-PSF FWHM mean and standard deviations from SExtractor (Bertin $\&$ Arnouts 1996) measurements of Tiny Tim simulations (Rhodes et al. 2007) of 2500 artificial stars in the GOODS field.

${ }^{\mathrm{c}}$ Nominal sky values for space-based observations.

Table 2 lists the mean and standard deviation of the 2500 PSF measurements for each of the four ACS filters. To account for the spread in these PSF distributions, each profile is modeled with three different values of the PSF FWHM: the nominal mean value and $\pm 5 \%$ of that value.

The GOODS ACS images are already sky subtracted, so no further attempt at measuring a residual sky was made. However, we do allow for sky subtraction errors in the decompositions, in a similar manner as the PSF errors, by using three different sky levels: the measured profile as is and at $\pm 0.5 \%$ of a nominal space-based value for each band. The adopted sky levels are listed in Table 2 .

The final step is to determine the best fit from the resulting 2160 decompositions. We follow M03 (their $\S 4.3$ ) and undertake a grid search for the minimum of two $\chi^{2}$ merit function distributions: the global $\chi^{2}$ (eq. [6]), which is dominated by the contribution from the disk, and a separate inner $\chi^{2}$ statistic computed to twice the radius where the bulge and disk contribute equally to the total luminosity $\left[r_{b=d} \equiv 2 r\left(I_{b}=I_{d}\right)\right]$. We label this statistic as $\chi_{\mathrm{in}}^{2}$. For cases where the bulges are so small that they never truly dominate the light profile (i.e., $r_{b=d}$ is undefined), we compute $\chi_{\text {in }}^{2}$ out to the radius at which $\nu=1$. Only the global $\chi^{2}$ is considered for single-component fits. Parameter $\chi_{\text {in }}^{2}$ was adopted to increase the sensitivity of the goodness-of-fit indicator to the bulge area. ${ }^{11}$

In cases where the bulge component is weak, fitting becomes more difficult, particularly where the rest-frame wavelength range extends into the UV for our highest $z$ galaxies. In order to avoid unrealistically large differences in the fits between the different bands, constraints were placed based on the $i$ - and $z$-band fits (where the SB profiles are closest to rest-frame $R$ band). These physical constraints are rather generous and do not contribute any subjective bias.

Figure 3 shows an example of the best-fit profiles and the fit residuals derived for all four bands of the $z=0.512$ spiral galaxy G928. The five structural fit parameters are shown in the upper right corner; the bottom panels show the run of ellipticity and PA from the isophotal fits. Note that these are the same for all four bands as the $z$-band fits are used for the profile extraction of the other three bands.

\subsection{Total Magnitudes and Spheroid Colors}

A key goal of our study is construction of the color-redshift relation for bulges in our sample. We thus explore in detail the

\footnotetext{
${ }^{11}$ Note that our algorithm minimizes the global $\chi^{2}$ only. The $\chi_{\text {in }}^{2}$ is calculated and used as a discriminator only after the algorithm has converged
} 

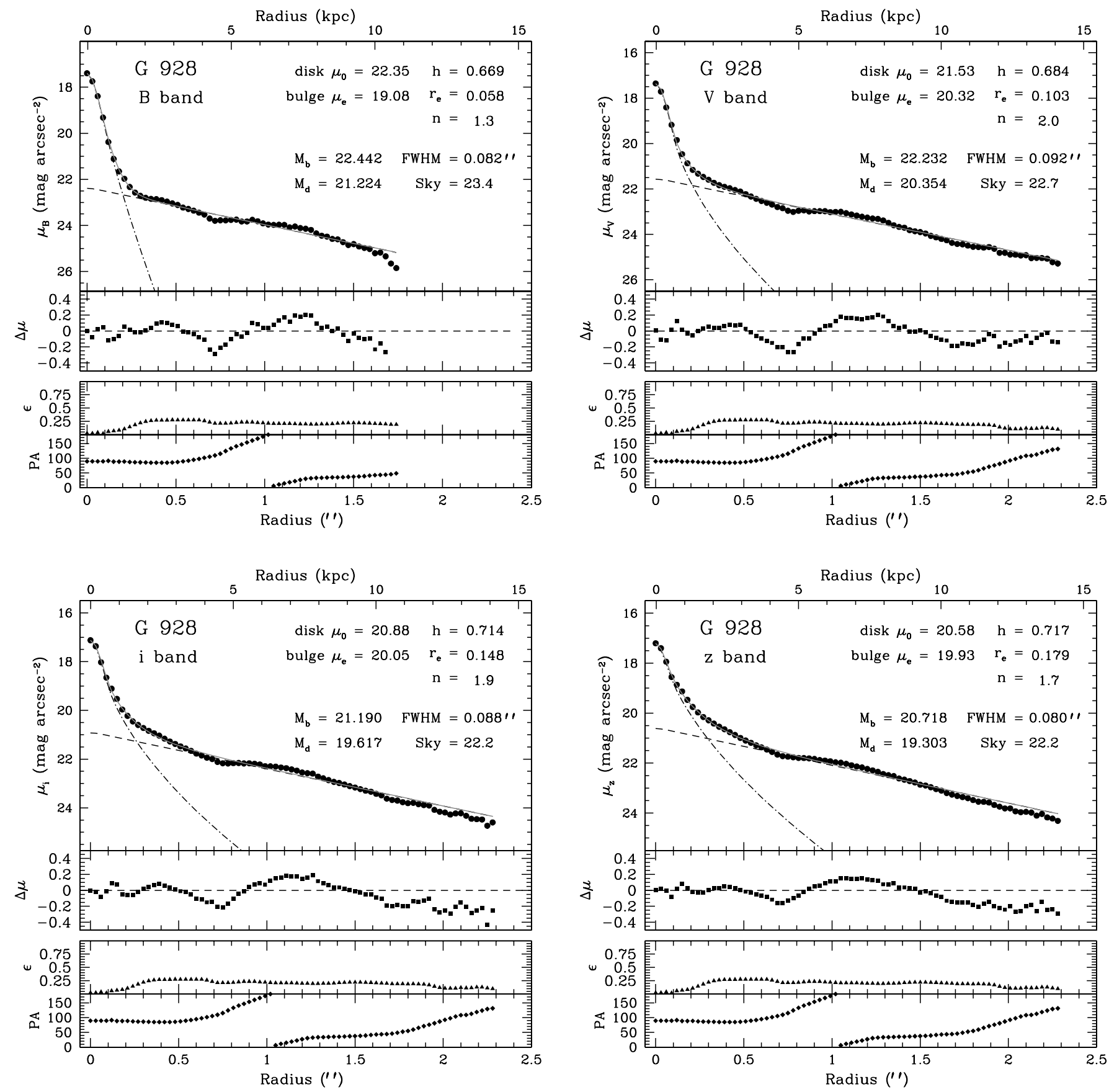

FIG. 3.-Example of $B / D$ decompositions for the $z=0.512$ spiral galaxy G928 in all four GOODS bands. In each figure, the top panel shows the measured SB profile ( filled circles), the bulge fit (dot-dashed line), the disk fit (dashed line), and the total bulge+disk fit (solid gray line). All fits are seeing-convolved using the best selected PSF values (see text). The five structural fit parameters are shown in the upper right corner, where the scales $\left(h\right.$ and $\left.r_{e}\right)$ are in arcseconds. The second panel shows the fit residuals where $\Delta \mu(r) \equiv$ fit $(r)$ - data $(r)$. The bottom two panels show the run of ellipticity and PA from the isophotal fits. Note that these are the same for all four bands as the $z$-band fits are used for the profile extraction of the other three bands. [See the electronic edition of the Journal for a color version of this figure.]

relationship between the colors of the spheroidal component and those determined using apertures. We likewise wish to derive integrated magnitudes so that we can more accurately determine the bulge/total ratios implied by our photometric analysis.

Magnitudes computed involving extrapolations will be highly sensitive to the actual profile shape. For example, outer disk (anti)truncations, which are often observed in spiral galaxies, would not be accounted for with a single exponential disk fit. We thus compute total galaxy magnitudes from the photometry to the maximum observed radius with the addition of the extrapolation of a linear fit to the outer $20 \%$ of the profile. The extrapolation typically increases the magnitude by $\simeq 0.1 \mathrm{mag}$, except when the profile is very shallow. In cases where the extrapolation adds more than $0.5 \mathrm{mag}$, the galaxy is tagged and the tabulated error is increased accordingly. We correct total magnitudes for (small) Galactic foreground extinction using the reddening values, $A_{\lambda}$, of Schlegel et al. (1998) and interpolating to the effective wavelength of the GOODS-ACS filters. The adopted values for both the northern and southern fields are listed in Table 2.

Similarly, the contribution to the total light at large radii (i.e., extrapolated to infinity) in the Sérsic profile changes significantly as a function of $n$ (see Fig. 2 of M03); thus, a small error on the fitted $n$ could lead to an error in the total magnitude. While this error will typically not be significant for the total spheroid 
magnitude, the relative differences between passbands can propagate to large errors on the colors whose dynamical ranges are small (of order $\sim 1.5 \mathrm{mag}$ for rest-frame $B-R$ and smaller for shorter wavelength baselines). To minimize the potentially large errors due to extrapolation, while still allowing for color gradients, we compute the colors of our fitted spheroids using the best-fit model parameters, but only integrating out to the radius corresponding to the best-fit $r_{e}$ in the $z$ band. These colors should be equivalent to aperture colors within $1 r_{e, z}$ for the single-component galaxies but can differ significantly from aperture colors for spiral bulges as they account for the (differential) contamination from disk light.

Bulge-to-total $(B / T)$ luminosity ratios can be computed either from the fits alone or as a combination of the bulge fit and the (nearly) nonparametrically measured galaxy magnitude discussed above. We adopt the latter approach as we have found that extrapolating a disk fit often overestimates its contribution (by $\sim 0.3 \mathrm{mag}$ on average).

\subsection{K-Correction}

The final step in deriving useful photometric quantities is the application of a $k$-correction to enable the compilation of restframe measures.

Often, a single function $K(z, X-Y)$, where $X-Y$ is the observed color in (optical) bands $X$ and $Y$, is derived and applied to entire samples, regardless of the galaxy type. For example, T05 provide transformations from the GOODS ACS filters to restframe Landolt $B$ and $V$ magnitudes (to within a few hundredths of a magnitude) for the redshift range $0 \leq z<1.25$. Two filters are used in each transformation depending on where the $4000 \AA$ break falls at the given redshift. Gebhardt et al. (2003) present a similar transformation, derived from 43 empirical spectral energy distribution (SED) templates, to convert from observed HST F606W and F814W magnitudes to rest-frame $U$ and $B$.

Alternatively, Blanton \& Roweis (2007) provide an IDL code, kcorrect, for computing $k$-corrections, which fits linear combinations of a set of model-based templates, including provision for emission lines and dust extinction, to the observed galaxy colors. The code kcorrect infers the underlying SEDs for galaxies over a range of redshifts by requiring that their SEDs be drawn from a similar population. The resulting reconstructed SEDs can then be used to synthesize the galaxy's rest-frame magnitude in any bandpass.

The different methods for deriving $k$-corrections will have their own set of advantages and drawbacks depending on the application. As a consistency check, in Figure 4 we compare the results from all three methods mentioned above. T05 provide transformations to rest-frame $B$ and $V$, while Gebhardt et al. (2003) give transformations to rest-frame $B$ and $U$. The comparison between the three methods gives reasonably consistent results for the $B$-and $V$-band $k$-corrections. The results are less consistent in the $U$ band. This is not surprising as it is more strongly dependent on the observed $B$-band magnitudes, which have the least well determined profiles. An additional investigation into the templates used in the kcorrect code implies that the dust extinction prescription employed also adds to this scatter. Regardless, we can be fairly confident in the absolute $B$ and $V$ rest-frame magnitudes derived from any of the three methods for the current sample. We adopt here the method of T05.

\subsection{Structural Parameters}

As a final check on our decompositions and sample characteristics, we examine here the structural parameters of our sample of spheroids with comparisons to local data. Figure 5 compares
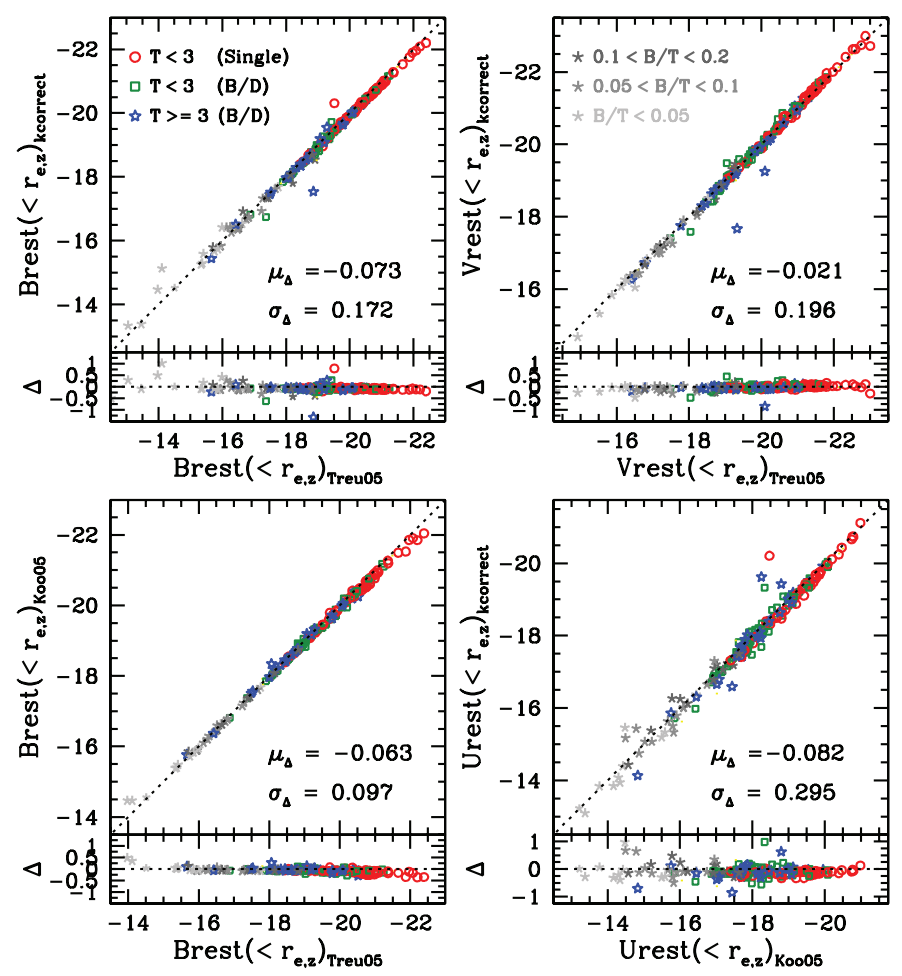

FIG. 4.-Comparison of rest-frame spheroid magnitudes within the $z$-band effective radius using three different methods for deriving $k$-corrections: T05, Gebhardt et al. (2003) (also provided by K05 and labeled as such in the figure), and kcorrect (Blanton \& Roweis 2007; see text for descriptions). Point types and colors (indicated in the upper left corners of the top panels) indicate the following: single-component spheroids (i.e., "pure" ellipticals; red circles); spheroid component of two-component systems with T type $<3$ ( $\equiv \mathrm{Sa}+\mathrm{b})$, i.e., the spheroid has been isolated from the disk via $B / D$ decomposition (green squares); and spheroid component of two-component systems with T type $\geq 3(\equiv \mathrm{Sa}+\mathrm{b})$ and $B / T>0.2$ (blue stars). Gray asterisks indicate bulges of galaxies with $B / T<0.2$, which are excluded from the FP analysis in $\S 6$. The bottom panel in each figure shows the residuals, and their respective mean and standard deviations are printed in the lower right corner of the top panels.

several correlations between our sample and the M03 sample of local bulges. Note that this local sample is heavily weighted toward low- $B / T$ late-type spirals whose bulges are, on average, best characterized by an exponential $(n=1)$ profile, and thus it does not cover the full parameter space of our higher $z$ sample. We restrict ourselves to this sample as the decompositions were done using the same method, and it is the characterization of the smallest bulges at higher $z$ that is most crucial to scrutinize. The reader is further advised that evolutionary effects (e.g., luminosity and/or size evolution) and selection effects (redshift-dependent limiting magnitude) have to be kept in mind when interpreting these correlations and comparing them to local samples.

Examination of Figure 5 reveals strong correlations between luminosity and both the effective radius (Fig. $5 c$ ) and Sérsic $n$ parameter (Fig. $5 d$ ). The correlation between luminosity and the effective SB within $R_{e}$ (Fig. $5 a$ ) is weaker, and it appears that the single-component Es have lower $\mathrm{SBe}$ for a given $M_{B}$ than the spiral bulges. Graham \& Guzmán (2003) point out that this is a manifestation of variations in profile shape as a function of total luminosity (shown in Fig. $5 d$; see also Fig. 12 of Graham \& Guzmán 2003). If one plots the central surface brightness of the bulge, $\mu(0)_{b}$, rather than $\mathrm{SBe}$, the relation becomes more linear with significantly less scatter over the full luminosity range.

Finally, Figure $5 b$ plots the so-called Kormendy (1977) relation between effective radius and SBe. At first glance, there does not appear to be any correlation. However, for the most luminous 

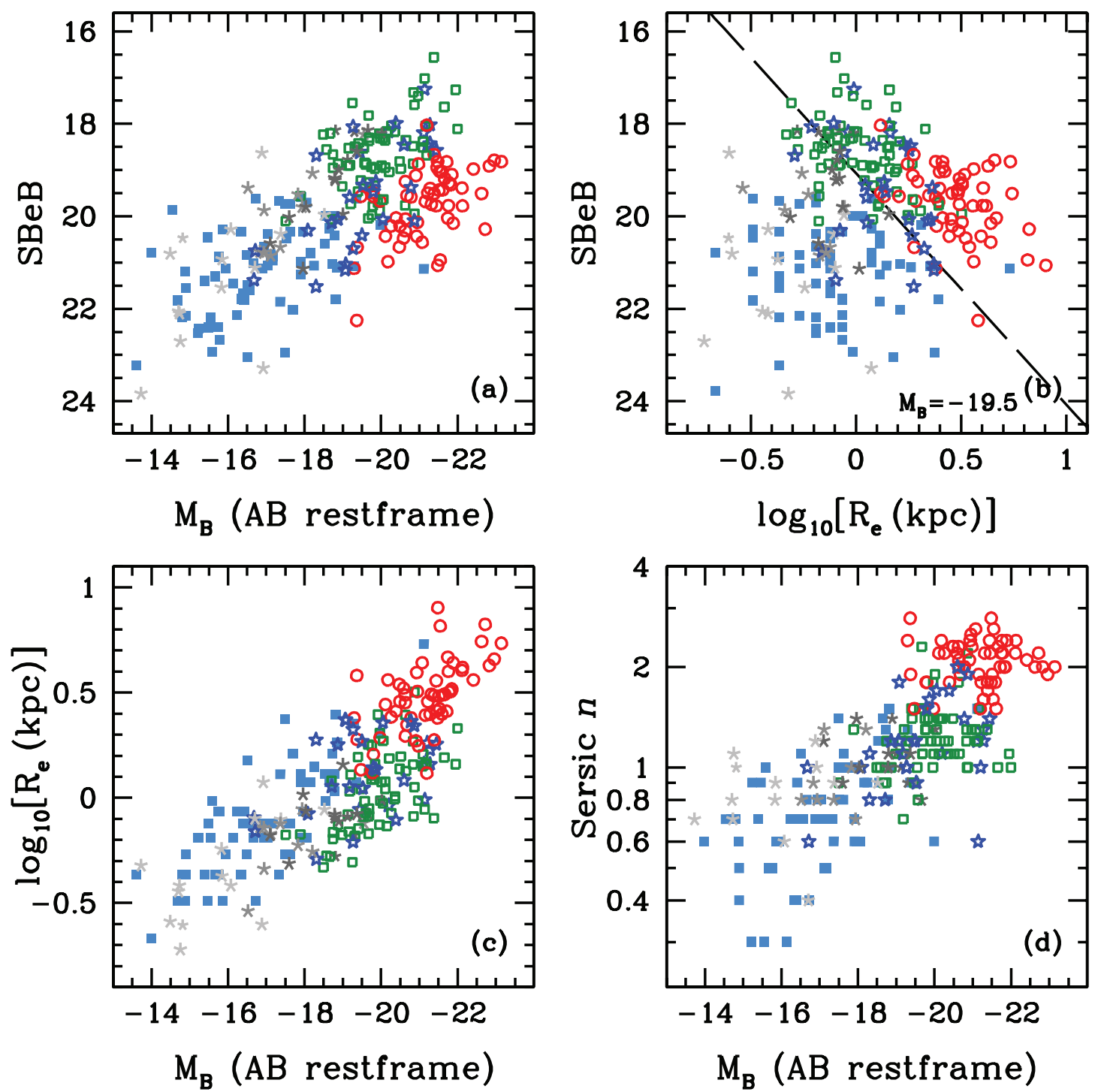

Fig. 5.-Correlation of photometric parameters for the spheroidal components of our intermediate-z galaxies (point types and colors are as in Fig. 4) and the local, mostly late-type, bulge sample of M03 (light blue squares). The dashed black line in $(b)$ is the line of constant magnitude $M_{B}=-19.5$ (see text for discussion).

spheroids with $M_{B}<-19.5$, lying above and/or to the right of the dashed line, which denotes a constant $M_{B}=-19.5$, a reasonably tight relation emerges. A linear fit to just the $M_{B}<-19.5$ spheroids gives a slope of 2.7, which is in rough agreement with previously determined values (e.g., La Barbera et al. [2003] find a constant slope of 2.9 for spheroidal galaxies out to $z=$ 0.64). The difference in slope and the large scatter observed here might be expected, given the broad redshift range. Fainter spheroids deviate from this relation having smaller $R_{e}$ and/or lower SBe. The transition at $M_{B}=-19.5$ roughly corresponds to the luminosity below which spheroid profiles are best characterized by a Sérsic $n \lesssim 1$ (exponential) shape. Thus, this represents the transition between cored $(n<1)$ and cuspy $(n>1)$ profile shapes.

Deviations in the Kormendy relation for smaller systems have been noted before in the context of differences between Es and dEs (e.g., Kormendy 1985). More recently this observation has extended to the bulges of local spiral galaxies in Ravikumar et al. (2006), who note that the fainter bulges appear more like the dEs, while the brightest bulges appear to follow the relation defined by the E/S0s. This also complies with the observation that both $\mathrm{dEs}$ and small bulges are more supported by rotation than their larger counterparts. The temptation to interpret the Kormendy relation as an evolutionary diagram or an indication of different formation mechanisms, however, has been disregarded due to the mixture between different types and the changes in the profile shapes discussed above.

Prior to our photometric analysis below, we summarize in Table 3 our derived photometric parameters for the spheroidal components of our sample galaxies.

\section{SPHEROID COLORS}

We now turn to the first component of the analysis of our data set, which is concerned with addressing the photometric evolution of our intermediate-redshift bulges. Both EAD01 and K05 presented color-redshift diagrams, but using different analysis methods and different sample selection criteria. Perhaps as a result of this, they arrived at rather different conclusions. Our goal is to understand the source of any discrepancy, as well as to intercompare the various approaches.

The dispersion in color at a given redshift is an important indicator of the diversity of the bulge population and hence the likelihood of continued SF and mass assembly. Moreover, a comparison with similar data for low-luminosity spheroidal galaxies 
TABLE 3

Spheroid Photometric Parameters

\begin{tabular}{|c|c|c|c|c|c|c|c|c|c|c|c|c|c|}
\hline $\begin{array}{l}\text { ID } \\
\text { (1) }\end{array}$ & $\begin{array}{l}n_{B} \\
(2)\end{array}$ & $\begin{array}{c}r_{e, B} \\
(\operatorname{arcsec}) \\
\quad(3)\end{array}$ & $\begin{array}{c}\mu_{e, B} \\
\left(\operatorname{mag~arcsec}^{-2}\right) \\
(4)\end{array}$ & $\begin{array}{l}n_{V} \\
(5)\end{array}$ & $\begin{array}{c}r_{e, V} \\
(\operatorname{arcsec}) \\
(6)\end{array}$ & $\begin{array}{c}\mu_{e, V} \\
\text { (7) }\end{array}$ & $\begin{array}{c}n_{i} \\
(8)\end{array}$ & $\begin{array}{c}r_{e, i} \\
(\operatorname{arcsec}) \\
(9)\end{array}$ & $\begin{array}{l}\mu_{e, i} \\
(10)\end{array}$ & $\begin{array}{c}n_{z} \\
(11)\end{array}$ & $\begin{array}{c}r_{e, z} \\
(\operatorname{arcsec}) \\
(12)\end{array}$ & $\begin{array}{l}\mu_{e, z} \\
(13)\end{array}$ & $\begin{array}{c}V-i \\
\left(<r_{e, z}\right) \\
(14)\end{array}$ \\
\hline $198 \ldots \ldots \ldots \ldots \ldots \ldots .$. & 0.9 & 0.31 & 23.95 & 1.3 & 0.34 & 22.42 & 1.4 & 0.32 & 21.34 & 1.4 & 0.33 & 21.08 & 1.04 \\
\hline $280 \ldots \ldots \ldots \ldots \ldots \ldots$ & 0.9 & 0.15 & 22.37 & 0.9 & 0.18 & 21.65 & 1.0 & 0.19 & 21.38 & 1.1 & 0.23 & 21.36 & 0.37 \\
\hline 319..................... & 0.9 & 0.08 & 23.57 & 0.8 & 0.11 & 22.05 & 0.9 & 0.13 & 21.43 & 0.9 & 0.14 & 21.32 & 0.84 \\
\hline $421 \ldots \ldots \ldots \ldots \ldots \ldots$ & 0.5 & 0.07 & 22.87 & 0.4 & 0.07 & 21.86 & 0.5 & 0.07 & 21.27 & 0.2 & 0.06 & 20.64 & 0.71 \\
\hline $438 \ldots \ldots \ldots \ldots \ldots \ldots \ldots$ & 0.1 & 0.01 & 22.13 & 0.9 & 0.11 & 22.71 & 1.0 & 0.13 & 22.19 & 1.3 & 0.20 & 22.37 & 0.90 \\
\hline $462 \ldots \ldots \ldots \ldots \ldots \ldots$ & 1.0 & 0.14 & 22.45 & 1.6 & 0.30 & 21.17 & 1.6 & 0.29 & 20.21 & 1.5 & 0.30 & 19.98 & 0.90 \\
\hline $465 \ldots \ldots \ldots \ldots \ldots \ldots$ & 1.2 & 0.20 & 24.03 & 1.0 & 0.16 & 21.74 & 0.9 & 0.16 & 20.71 & 0.9 & 0.16 & 20.57 & 0.94 \\
\hline
\end{tabular}

Notes.-Col. (1): Internal galaxy ID. Cols. (2)-(4): Sérsic $n$ shape parameter, effective radius, and effective surface brightness (uncorrected), respectively, from the light profile modeling (see $\S 3.1$ ) measured in the GOODS-ACS $B$-band (F435W) filter. Cols. (5)-(7): Same as cols. (2)-(4), but for the GOODS-ACS $V$-band (F606W) filter. Cols. (8) - (10): Same as cols. (2)-(4), but for the GOODS-ACS $i$-band (F775W) filter. Cols. (11)-(13): Same as cols. (2)-(4), but for the GOODS-ACS $z$-band (F850LP) filter. Col. (14): Observed $V-i$ color measured out to $1 r_{e}$ (see $\S 4$ ). Table 3 is published in its entirety in the electronic edition of the Astrophysical Journal. A portion is shown here for guidance regarding its form and content.

will give an indication of the extent to which growth is primarily driven by processes internal to spiral galaxies.

EAD01 based their analysis on aperture colors (within a fixed size of 5\% relative to the isophotal radius) and found that their bulges displayed a large scatter toward the blue in observed $V-I$, with few being as red as E/S0 counterparts at a given redshift. They concluded that bulges underwent recent periods of growth and associated rejuvenation. However, it is possible that the blueward scatter arises, in part, from disk contamination. As we have undertaken careful decomposition of the light profiles with the improved ACS data, here we examine the equivalent color trends with redshift for our sample.

\subsection{Comparison with Aperture Colors}

Bulge colors computed from $B / D$ decompositions are extremely sensitive to the fits themselves. To understand some of these effects, and to calibrate some of the contamination that might have affected the EAD01 analysis, in Figure 6 we compare our fitted bulge $V-i$ colors (measured out to $1 r_{e}$ ) to the central $0.3^{\prime \prime}$ aper- ture colors (left panel) and those of the total galaxy (right panel). The ACS PSF is $\lesssim 0.09^{\prime \prime}$, so the $0.3^{\prime \prime}$ aperture colors should not be seriously affected by PSF mismatch between bands [which are $\left.\Delta(\mathrm{PSF}) \lesssim 0.008^{\prime \prime}\right]$. Bulge fits are not shown if the fit magnitudes in either band were fainter than $30 \mathrm{mag}$ (as these galaxies are fundamentally bulgeless).

The fit and aperture $V-i$ colors are very similar, but there is some scatter such that the model fit colors are often somewhat redder than the aperture colors, particularly for galaxies that have a disk component (squares, stars, and asterisks). This probably arises from (bluer) disk contamination within the aperture. The fit colors are also often redder than the total galaxy, consistent with negative (bluing outward) gradients. Nonetheless, the above comparison confirms that our photometric decomposition is stable and yields reasonably consistent bulge colors.

\subsection{Colors versus Redshift}

We now return to the redshift dependence of our observed spheroidal component colors. Figure 7 plots the observed $V-i$
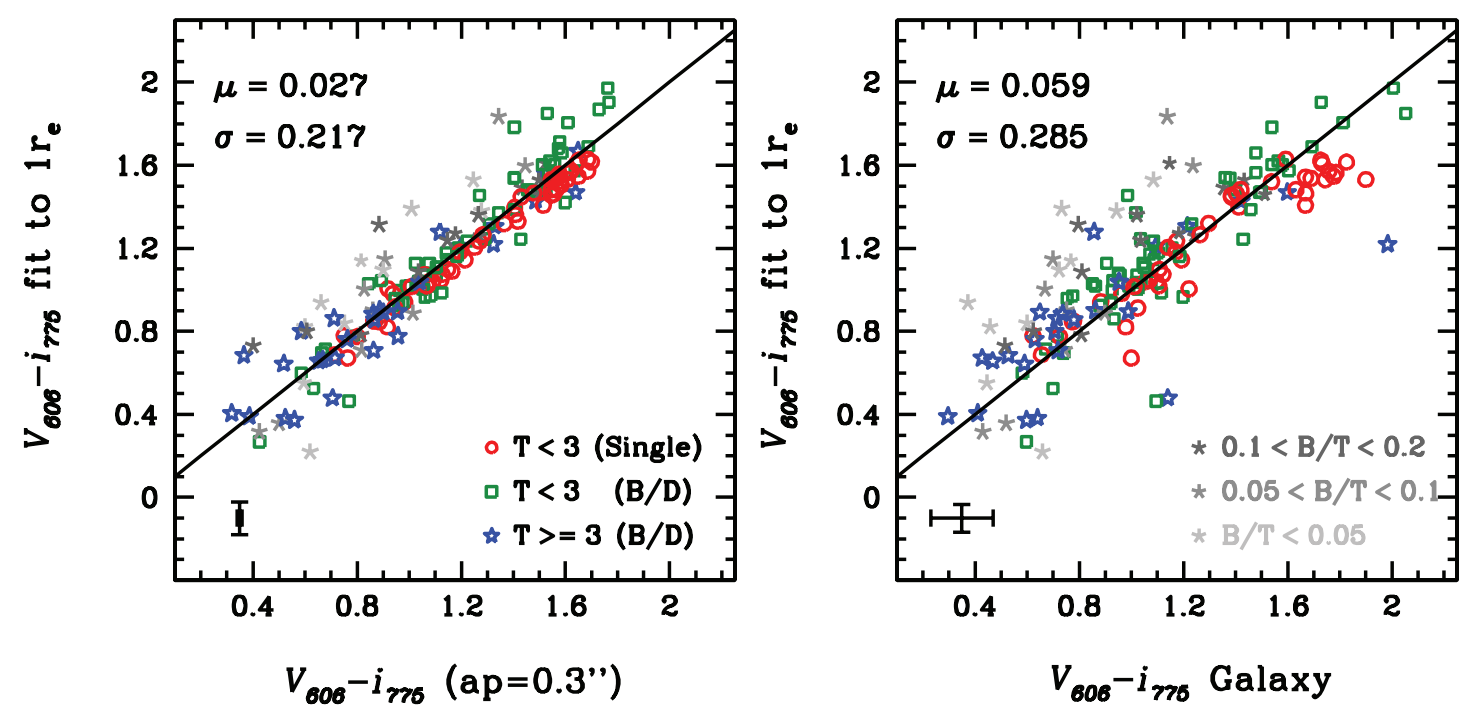

FIG. 6.-Comparison of observed F606W - F775W $(V-i)$ color from the model fits with the $0.3^{\prime \prime}$ aperture color (left) and total galaxy color ( right). Point types and colors are indicated (lower right corner), and the average errors are shown (lower left corner). The mean difference and standard deviation are given (upper left corner), and the solid line is the one-to-one relation. 


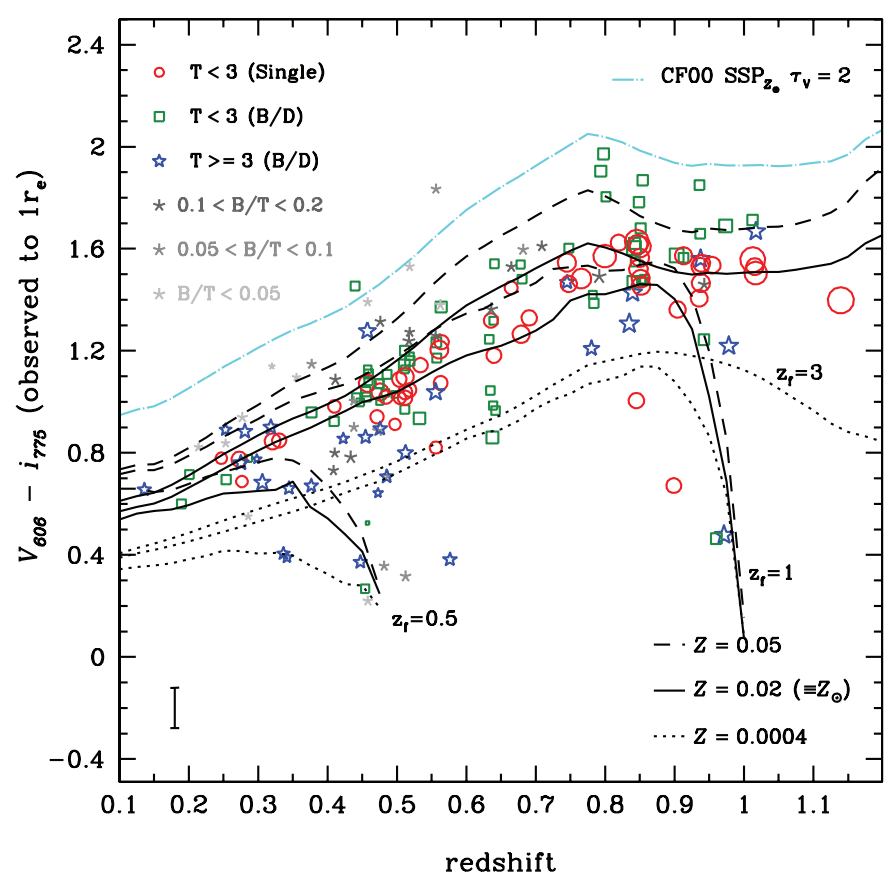

FIG. 7.- Observed $V-i$ spheroid color (measured to $1 r_{e}$ from the light profile modeling) vs. redshift. Point types and colors are indicated in the upper left corner, and the point size is proportional to the rest-frame $B$ magnitude of the spheroidal component. The black lines represent BC03 models of single-burst stellar populations formed at three redshifts $\left(z_{f}=0.5,1,3\right)$ that evolve passively. Single-burst stellar populations for three metallicities are shown $(Z=0.0004,0.02,0.05$, where $\left.Z_{\odot}=0.02\right)$. The cyan dot-dashed line is a $z_{f}=3$ model with solar metallicity that has extinction included using the dust models of Charlot \& Fall (2000) and $\tau_{V}=2$. The average error is shown as the error bar (lower left corner).

colors as a function of redshift. The black lines represent model tracks of a passively evolving single-burst population for three formation redshifts $\left(z_{f}=0.5,1,3\right)$ and three metallicities $(Z=$ $0.004,0.02,0.05)$. All stellar population models considered are those of Bruzual \& Charlot (2003, hereafter BC03). The dotdashed cyan line is a $z_{f}=3$ model with solar metallicity that has extinction included using the dust models of Charlot \& Fall (2000) with $\tau_{V}=2$ (corresponding to an $A_{B} \sim 1.5$ ).

As observed by many authors (e.g., T05), most of the elliptical (i.e., single-component) galaxies (circles) are consistent with a single-burst model with $z_{f} \simeq 3$. Those Es that deviate from this trend, indicating a more recent formation/activity, tend to be the lower luminosity systems (as indicated by the point size). Many of the two-component galaxies (squares, stars, and asterisks) also follow this trend, but a more significant number scatter strongly to the blue and a few lie redward of the dust-free models (even if a very high metallicity is assumed). The latter may arise from dust extinction, but the large scatter to the blue is consistent with the observations of EAD01 (see their Fig. 4). However, unlike EAD01, we do see a number of bulges that are just as red as the E/S0s. These could represent the red bulges observed by K05, and, indeed, if a similar cut is made on the observed $i$-band bulge magnitude as that of $\mathrm{K} 05$, the remaining spheroids in the same redshift range $(z \sim 0.8)$ are red and consistent with old singleburst populations.

Thus, it seems that there is no real disagreement between the studies of EAD01 and K05. While there are differences in their analysis techniques, our own internal tests suggest that these are unlikely to be significant to a level that could cause such a discrepancy $(\S 4.1)$. The fundamental difference is in the respective bulge samples: EAD01 sampled bulges drawn from a full range of Hubble types with the morphologically classed spirals likely skewed toward later type bulges. K05 selected bulges only from the bright end of the bulge luminosity function, which, according to Figure 7, are predominantly red and passively evolving.

In summary, therefore, our results confirm the diversity of bulge colors at intermediate redshift originally identified by EAD01, although the bulk of the more luminous systems more closely track the relationship defined by the larger spheroidal galaxies. The question thus arises whether we are looking at two different populations each following a distinct formation path, or rather a more continuous mode of assembly for the spheroidal components where, for example, additional growth is occurring but it has proportionally less effect for the more massive systems.

Phrased another way, we can ask whether the spheroidal components of all galaxies (spirals, S0s, and Es) at a given mass are consistent in terms of their evolutionary paths. It is hard to address such a question from colors alone as the bulge luminosity and $B / T$ ratio represent poor proxies for the mass of the system. To address this more fundamental question, we now turn to considering the combined set of dynamical and photometric information for our sample. By constructing the FP for a subset of our bulge components and comparing the relation to that now well mapped for spheroidal galaxies of various masses (T05), we can more accurately determine the mass growth rate of luminous bulges.

\section{SPECTROSCOPIC DATA AND ANALYSIS}

Our Keck DEIMOS spectroscopic data are used to derive central stellar velocity dispersions that, together with the photometric parameters derived in $\S 3$, enable us to construct the FP for the spheroidal components of E, S0, and spiral galaxies and to compare their recent stellar mass growth rates.

As discussed earlier, the spectroscopic data were taken in two distinct campaigns. The first is that described in detail in T05, relating to both $\mathrm{E} / \mathrm{S} 0$ s and bulge-dominated spirals. The second campaign was designed to increase the sample of spiral bulges for the current study, particularly in the redshift range $0.1<z<$ 0.7 . Targets were selected with $\mathrm{Sa}+\mathrm{b}(\mathrm{T}=3)$ morphologies from the Bundy et al. (2005) catalog to a limit of $z_{\mathrm{AB}}^{\prime}=22.5$, corresponding to spirals with luminosities $-18.5<M_{B}<-20.5$.

In the following sections we describe how the new spectroscopic observations were undertaken and discuss the extraction of central velocity dispersions in the context of the earlier work by $\mathrm{T} 05$.

\subsection{Observations and Data Reduction}

The spectroscopic observations used here were taken with DEIMOS on the Keck II Telescope. The data set comprises a total exposure time of 5-7 hr for each of four masks (three in the northern GOODS field, one in the southern GOODS field) spanning three observing campaigns in 2004 March, 2004 December, and 2005 March. A further campaign on GOODS-S in 2005 October was unsuccessful due to bad weather, and yet another campaign scheduled in 2006 October was canceled due to a major earthquake that ceased all observatory operations. Most of the data were obtained in seeing conditions in the range of $0.8^{\prime \prime}-1.0^{\prime \prime}$ (FWHM). Tilted slits were used and aligned along the major axis wherever possible (for DEIMOS masks, slits can only be tilted up to $30^{\circ}$ from the mask PA).

We used the 1200 line gold-coated grating blazed at $7500 \AA$ and centered at $8000 \AA$, which offers high throughput in the red, a pixel scale of $0.1185^{\prime \prime} \times 0.33 \AA$, and a typical wavelength coverage of $2600 \AA$. A resolution of $\sim 30 \mathrm{~km} \mathrm{~s}^{-1}$ was determined from arc lines and sky emission lines for the $1^{\prime \prime}$ wide slitlets. In 

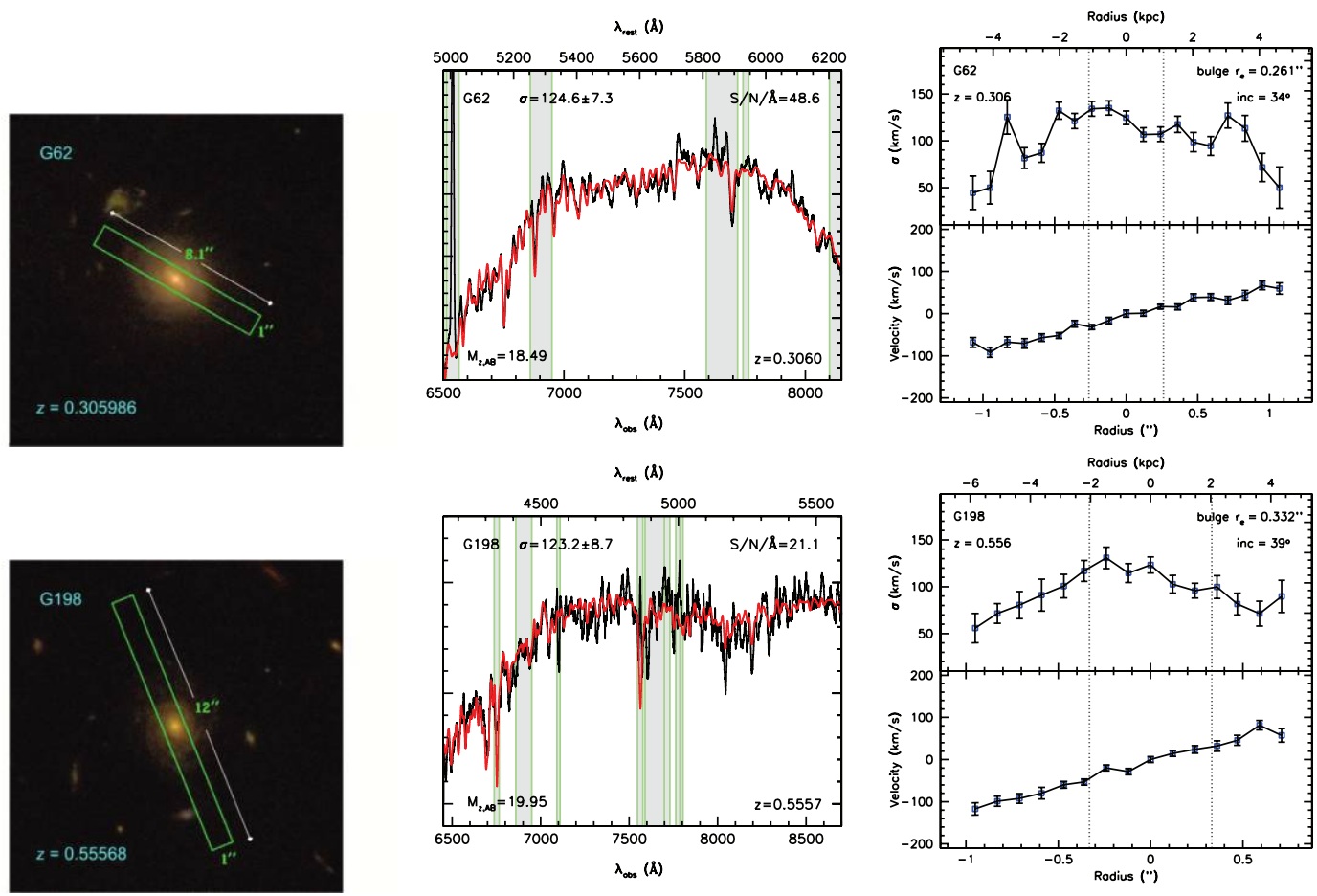

FIG. 8.-Spectroscopic measurements for all galaxies from the new bulge sample with $B / T>0.2$. Left panels: Three-color ACS image and slit orientation. Middle panels: Observed central galaxy spectrum (black lines) and the best-fitting stellar template (red lines) convolved to the measured velocity dispersion. Shaded regions (flanked by vertical green lines) indicate regions masked out during the fit due to an overlap with either A and B atmospheric absorption bands, emission lines (Balmer and $[\mathrm{O}$ III] $\lambda \lambda 5007,4959$ in particular), bad regions in the stellar templates, or the gap between the blue and red spectra. The spectra have been smoothed with a $10 \AA$ boxcar filter. Galaxy ID, $\sigma$ (in $\mathrm{km} \mathrm{s}^{-1}$ ), S/N/Å of the observation, observed $z$ magnitude, and redshift are indicated. Right panels: Kinematic profiles. Top: Velocity

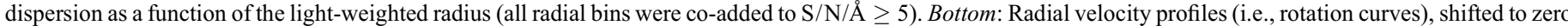
velocity at the center (determined from the peak of the spectroscopic light profile). Solid black lines connect the points to guide the eye. The vertical dashed lines are located at $\pm 1 r_{e}$ as determined by the $B / D$ decompositions.

deriving velocity dispersions, the resolution was measured independently for each slit as discussed below.

The raw spectra were reduced using the DEIMOS reduction pipeline as developed by the DEEP2 Redshift Survey Team (Davis et al. 2003). After extracting 1D sky-subtracted spectra, we estimated the redshift and spectral type for each galaxy. Zspec, an IDL-based software package also written by the DEEP2 Team, was used to evaluate the accuracy of the assigned redshift, and a quality criterion $q$ ( 1 for stars, 2 for possible, 3 for likely, and 4 for certain) was assigned. All spectra in our sample have a redshift quality $\geq 3$, and most have $q=4$.

Noting that the minimum $\mathrm{S} / \mathrm{N} / \AA \AA$ for accurate central dispersions (hereafter $\sigma_{0}$ ) is $\gtrsim 10$ (see, e.g., Treu et al. 2001), coupled with the desire to extract radial kinematic profiles, the spectra were co-added in radial bins to ensure a minimum S/N/Â of 5 . Failure to obtain accurate $\sigma_{0}$ measurements occurred due to low$\mathrm{S} / \mathrm{N}$ spectra, bad sky subtraction, unfortunately placed absorption features with respect to the atmospheric $\mathrm{A}$ and $\mathrm{B}$ absorption bands, or bulges whose velocity dispersions probably lie below our resolution limit $\left(\sim 30 \mathrm{~km} \mathrm{~s}^{-1}\right)$. We detail below our methodology for deriving these dispersions.

\subsection{Measuring Velocity Dispersions}

The velocity dispersion and rotation profiles of our spectra were measured using the well-tested Gauss-Hermite pixel fitting algorithm provided by van der Marel (1994). ${ }^{12}$

Prior to analysis, the 1D galaxy and template spectra are matched in rest-frame wavelength range and rebinned onto a logarithmic

\footnotetext{
12 Obtained at http://www-int.stsci.edu/ marel/software/.
}

wavelength scale. After convolving a suitable template spectrum with Gaussian velocity profiles of various dispersions, the bestfitting dispersion for each template is found (see Fig. 8, middle panels). The goodness of fit is measured via the $\chi^{2}$ statistic, weighted by the observational errors. The parameters determined for a given spectrum include the model-to-galaxy relative line strength $(\gamma)$, the mean velocity $(v)$, the stellar velocity dispersion $(\sigma)$, and their respective errors. An advantage of the pixel fitting technique is the ability to mask, via zero weighting, undesirable regions of the spectrum, for example, those containing emission lines, gaps between CCDs, and atmospheric features.

Ideally, the template spectra should be an excellent match to the spheroid stellar population (González 1993). From our previous analysis of GOODS E/S0s (T05), we have an appropriate set of high-resolution stellar templates that range from spectral types G0 III to K5 III. Recognizing that one of the goals of this study is to address the conjecture that bulges contain younger stellar populations, potentially spanning a range of metallicities, in addition to the observed red giant templates, we also include a selection of 36 templates from the high-resolution synthetic spectra of Coelho et al. $(2005)^{13}$ spanning the range $T_{\text {eff }}=4000-$ $7000 \mathrm{~K}, \log _{10}(g)=15-45$, and $[\mathrm{Fe} / \mathrm{H}]=-2.0$ to +0.5 . The best-fit single stellar or model template is selected primarily on the $\chi^{2}$ figure of merit, but some weight is given to the line strength parameter $\gamma$ (which should equal unity for a perfect spectral match between galaxy and template) and the formal error of the fit parameters. Indeed, in many cases, the bulge spectra are best matched by the "younger" model template spectra.

${ }^{13}$ Obtained at http://www.mpa-garching.mpg.de/PUBLICATIONS/DATA/ SYNTHSTELLIB/synthetic_stellar_spectra.html. 

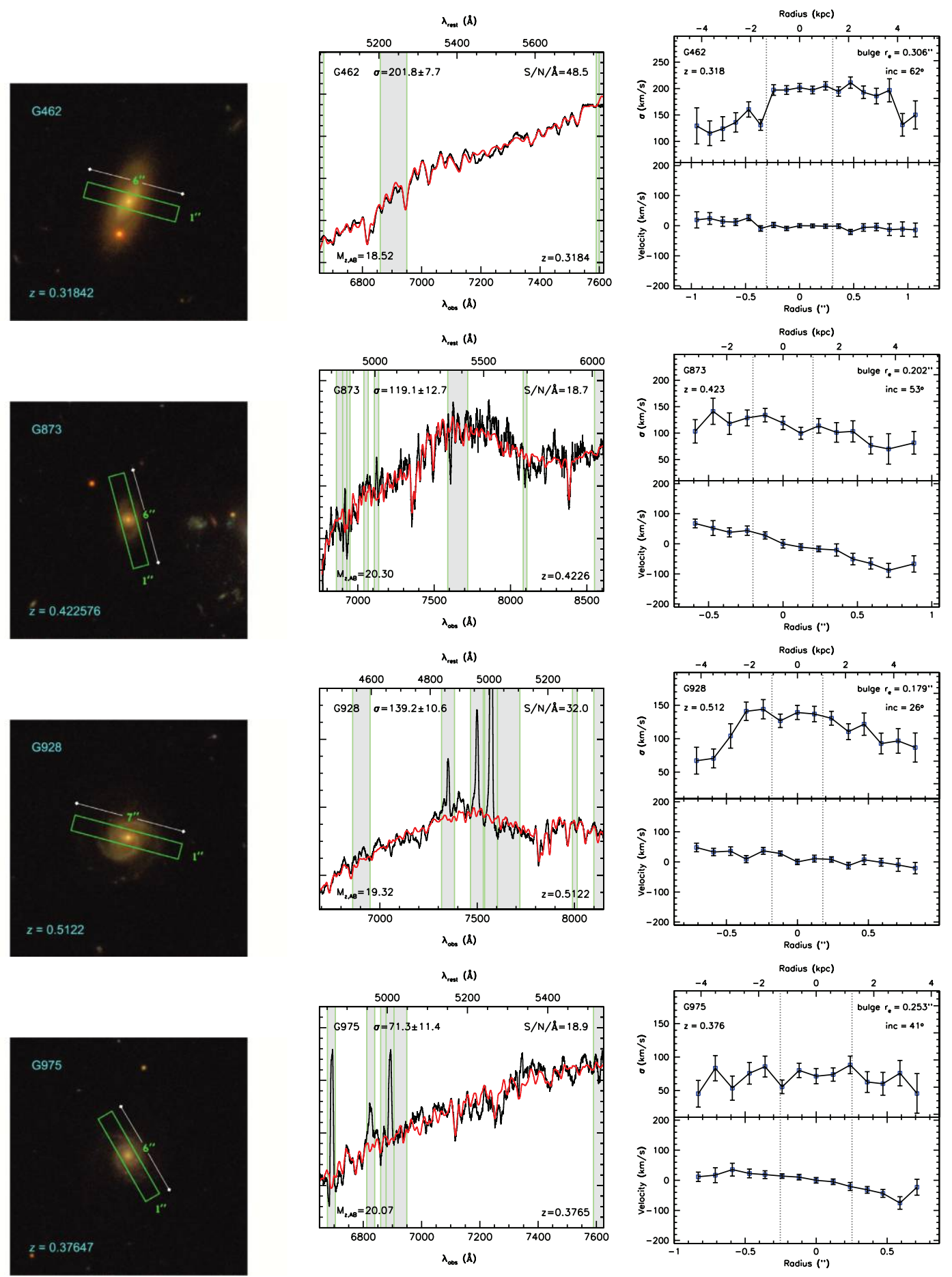

FIG. 8-Continued

Out of 45 spiral bulges observed in this second observing campaign, successful central dispersions were obtained for 23 galaxies; 7 had marginal measurements, 13 failed to yield dispersions, and 2 had corrupt spectra. Of the 23 galaxies with successful $\sigma_{0}$ measurements, only 8 had $B / T>0.2$ and thus could be included in our FP sample. The velocity dispersion fits for these eight galaxies are shown in Figure 8 (middle panels).

Finally, of the eight galaxies observed in common in both campaigns, five had successful $\sigma$ measurements in both and can be compared as a consistency check. Four of the galaxies had $\delta \sigma / \sigma<0.08$ with no systematic trend. The fifth galaxy (G928) had $\delta \sigma / \sigma=0.54$, but this is not surprising nor worrisome, given that the spectrum is dominated by young stellar populations and emission lines, while the measurement procedure in T05 was only appropriate for older stellar populations, starting with the choice of red giant stellar templates. We thus conclude that the measurements are consistent within the error bars, when they are comparable.

\subsection{Aperture Corrections and Disk Contamination}

Disk contamination could potentially present a source of uncertainty when interpreting the central velocity dispersions for 

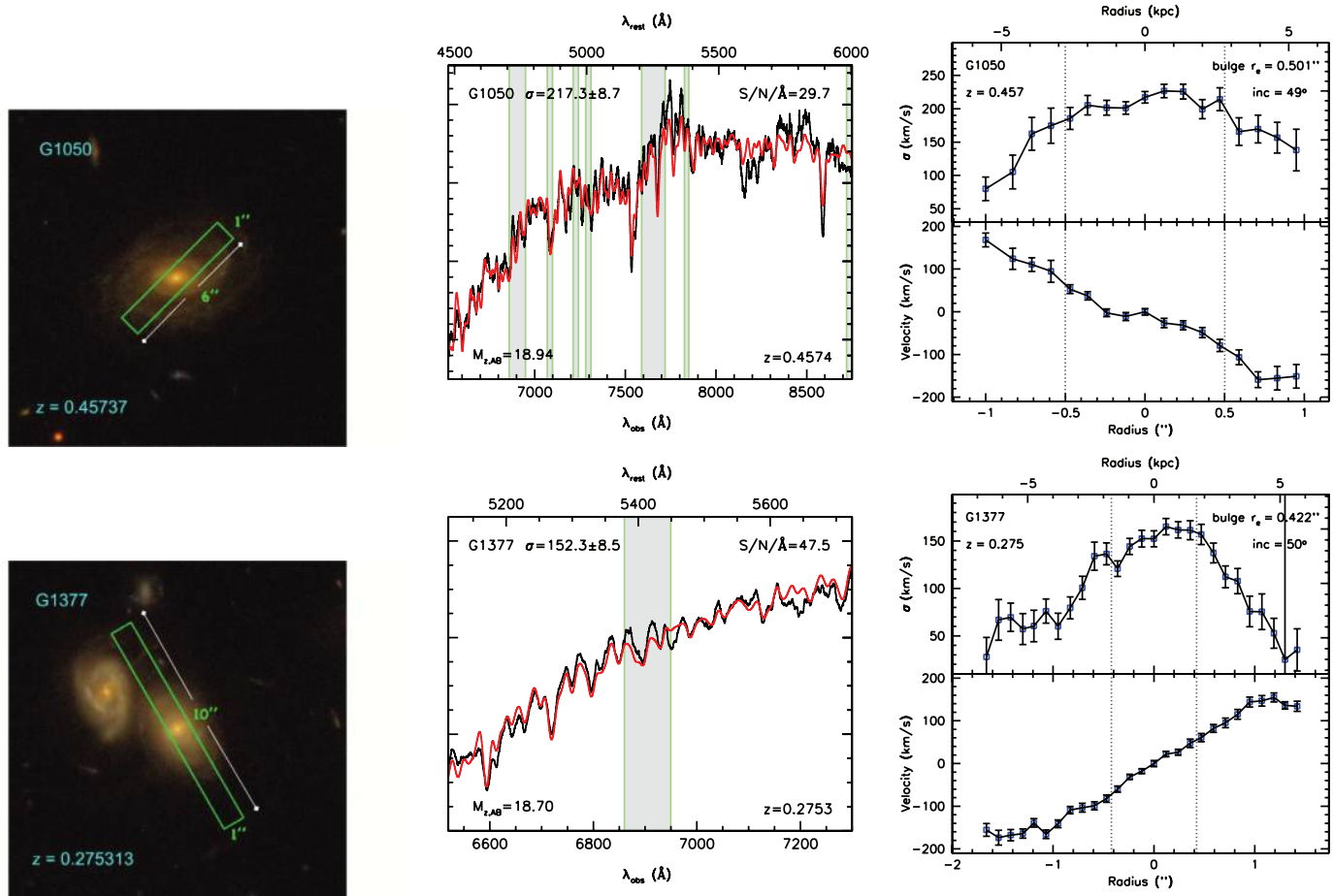

FIG. 8-Continued

these spiral galaxies as representative of the bulge component. In addition to template mismatch biases, even a modest systematic rotation might lead to an overestimated value for the smaller bulges. The spatial resolution of the observations is limited by the pixel scale $\left(0.1185^{\prime \prime}\right.$ pixel $\left.^{-1}\right)$, the physical size of the slit width $\left(1^{\prime \prime}\right)$, and the typical seeing FWHM during the time of the observations $\left(\sim 0.8^{\prime \prime}-1^{\prime \prime}\right)$. As mentioned in $\S 2$, we have limited our bulge sample to those with $B / T>0.2$ to ensure that the bulge light dominates in the center. However, the presence of a disk could still influence the measurement and interpretation of $\sigma_{0}$.

A proper kinematic decomposition of our data into contributions from the bulge, disk, and dark halo components would require full dynamical modeling (see Widrow \& Dubinski 2005). Conceivably this could be possible for a few galaxies where we have adequately sampled kinematic profiles, but it is beyond the scope of the current analysis. Our main goal is therefore to estimate the likely degree to which disk light is influencing our measurements.

For those spectra where resolved kinematic data can be extracted (see Fig. 8, right panels), we can attempt to estimate the disk contribution to the true bulge dispersion, $\sigma_{0, b}$, from the rotation measured across the bulge (assuming that this arises entirely from the disk component). We model this as

$$
\sigma_{0, b}=\sqrt{\sigma_{0}^{2}-\left[V\left(r_{\mathrm{ap}}\right) / \sin i\right]^{2}},
$$

where $V\left(r_{\mathrm{ap}}\right)$ is the velocity at $1 r_{e}$ or at a radius of 2 pixels (at $0.1185^{\prime \prime}$ pixel $^{-1}$ ), whichever was larger. The velocity was typically very small, with the correction factor $\sigma_{0, b} / \sigma_{0}$ ranging from 0.7 to 1 , with a mean value of 0.95 . We can also monitor the possible effects of disk contamination in the bulge spectra by considering the inner dispersion gradients. Inspection of Figure 8 (right panels) reveals that the $\sigma(r)$ profiles are largely flat within the central regions $\left(\lesssim 1 r_{e}\right)$. Although we do not have kinematic profiles for all galaxies in the combined sample, given that the bulk have near face-on inclinations, we conclude that contamination by a rotating disk component is not significant.

Finally, we need to standardize the aperture to which the dispersion measurement refers. Several studies have considered $\sigma$ gradients in early-type galaxies and find $\sigma_{r} / \sigma_{r_{e}}=\left(r / r_{e}\right)^{\alpha}$, with $\alpha=-0.04$ to -0.06 (e.g., J96; Cappellari et al. 2006). For our observations, we have a fixed slit width of $1^{\prime \prime}$, the DEIMOS scale is $0.1185^{\prime \prime}$ pixel $^{-1}$, and the effective radii of our galaxies range from $0^{\prime \prime}$ to $1^{\prime \prime}$, with an average value of $0.3^{\prime \prime}$. As a result, although our extracted central spectrum will be dominated by the highest SB central portion, there will be some contribution from beyond $1 r_{e}-2 r_{e}$. Adopting an observed effective aperture of $0.35^{\prime \prime}$, the average correction to an $r_{e} / 8$ aperture using $\alpha=-0.06$ is $\sigma_{r_{e} / 8}=$ $1.16 \sigma_{\mathrm{ap}}$, where we have chosen the standard correction to an aperture of $r_{e} / 8$ for comparison of our results with other studies. Adopting $\alpha=-0.04$ would reduce the correction factor to 1.10 . Our correction takes into account the measured $r_{e}$, which has the drawback of correlating $\sigma$ and $r_{e}$ but is important given the wide range of spheroid sizes in our sample relative to the constant effective aperture of our observations.

\subsection{Fundamental Plane Parameters}

We now use the derived photometric and kinematic parameters to construct the FP for our intermediate- $z$ sample of the spheroidal components of galaxies with $B / T>0.2$. The FP is traditionally expressed in terms of galaxy effective radius, $R_{e}$, the average surface brightness within the effective radius, $\mathrm{SBe}$, and the central velocity dispersion, $\sigma_{0}$ (e.g., Dressler et al. 1987; Djorgovski \& Davis 1987), related via

$$
\log _{10}\left(R_{e}\right)=\alpha \log _{10}\left(\sigma_{0}\right)+\beta \mathrm{SBe}+\gamma,
$$

with $R_{e}$ in units of kpc, SBe in mag $\operatorname{arcsec}^{-2}$, and $\sigma_{0}$ in $\mathrm{km} \mathrm{s}^{-1}$.

In order to address the evolutionary history of mass buildup in galaxies, it is necessary to convert these measured parameters into masses and mass-to-light $(M / L)$ ratios. An effective dynamical 
estimate of the stellar mass, $M$, can be defined using the scalar virial theorem for a stationary stellar system as

$$
M \equiv \frac{c_{2}(n) \sigma_{0}^{2} R_{e}}{G},
$$

where $c_{2}(n)$ is the virial coefficient. In the case of structural homology, the virial coefficient is a constant for all galaxies; thus, the FP maps directly into an $M / L$ ratio (see, e.g., T05) using the usual definition for luminosity,

$$
L \equiv 2 \pi\left\langle I_{e}\right\rangle R_{e}^{2}
$$

where $\left\langle I_{e}\right\rangle$, the average intensity within the effective radius, $R_{e}$, is given in equation (5). However, in the case of varying $n$, the profile shape does affect the measured $M / L$ through a variation in the velocity dispersion profile (even though these changes in profile shape do not significantly alter the FP or its tilt; see Fig. 9).

A number of authors have constructed spherical dynamical models to derive $c_{2}(n)$ for different profile shapes (e.g., Ciotti et al. 1996; Prugniel \& Simien 1997; Bertin et al. 2002; T04), with generally consistent results. We use the derivation of T04, who constructed nonrotating isotropic spherical models, and take into account the projected velocity dispersion over an effective aperture. ${ }^{14}$

For the purpose of assessing evolutionary trends, we need a local relation (at $z=0$ ) against which we can compare higher $z$ galaxies. Ideally, one would like to have a local comparison sample that is large in numbers and homogeneous in measurement techniques to the distant sample. We are currently undergoing a major observational project at the Palomar Observatory to construct such a large, homogeneous, local sample that extends to spiral galaxies and includes both field and cluster samples (L. A. MacArthur et al. 2008, in preparation).

While we await the results from that campaign, we derive a local FP comparison from a sample of early-type galaxies in the Virgo Cluster. We start from the structural analysis of F06 of HST images of early-type galaxies from the ACS Virgo Cluster Survey (ACSVCS) of Côté et al. (2004). Precise distance measurements come from the surface brightness fluctuation analysis of Mei et al. (2007), and velocity dispersions are carefully collected from the literature. A detailed description of our derivation of the local FP relation for Virgo Cluster early-type galaxies, which relaxes the assumption of structural homology, is provided in the Appendix. The result is

$$
\log _{10}\left(M / L_{B}\right)_{0}=0.225 \log _{10}(M)-1.63,
$$

and we measure the offset for galaxy $i$ from this local relation as

$$
\Delta \log _{10}(M / L)^{i}=\log _{10}(M / L)^{i}-\log _{10}(M / L)_{0}^{i} .
$$

We note that our local relation is poorly constrained at present, especially at the high-mass end, largely due to a dearth of massive objects in our pruned local sample (see the Appendix).

Finally, since we have not applied the usual formalism found in the literature of imposing a fixed profile shape to parameterize the light profiles, some care in the analysis and, in particular, its comparison with previous works must be taken. The correlation between the Sérsic $n$ and total luminosity seen in Figure $5 d$ indicates weak homology among the spheroidal components of galaxies, such that spheroids of a given luminosity have the same

\footnotetext{
14 Note that models based on a single Sérsic profile, such as T04, neglect the effects of a dark matter component, which could be important to understand other aspects of the tilt of the FP (see Bolton et al. 2007).
}

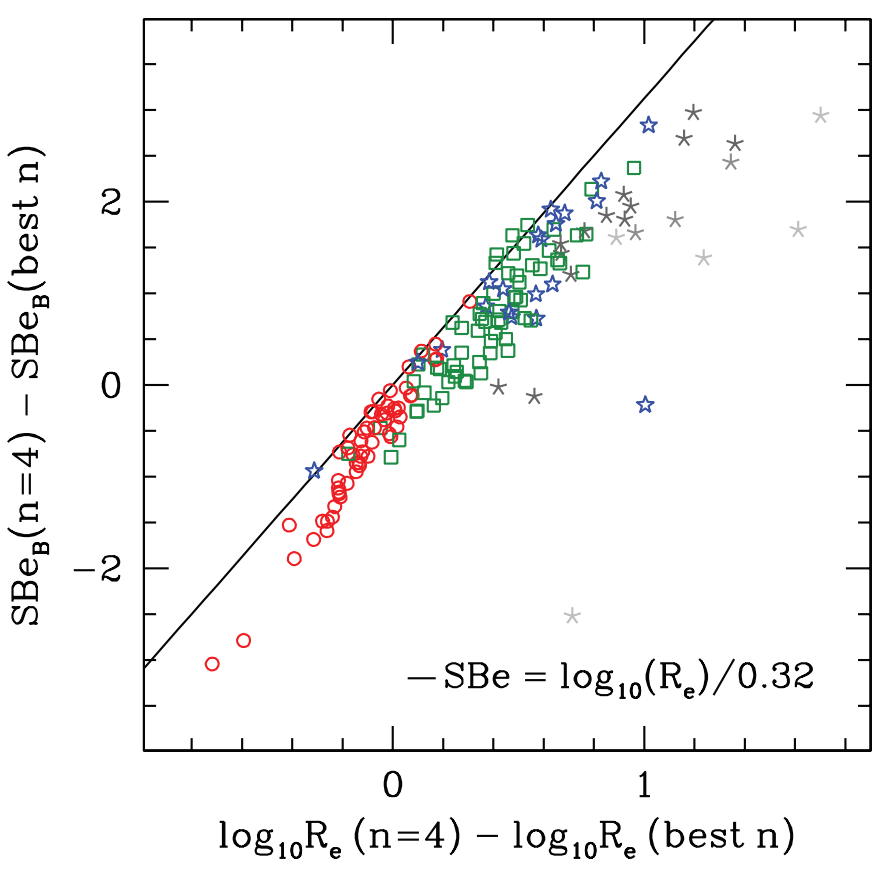

FIG. 9.-Comparison of Sérsic fit parameters derived here with the T05 fixed de Vaucouleurs fits shown as the difference in SBe vs. the difference in $\log _{10}\left(R_{e}\right)$. Point types and colors are as in Fig. 4. The solid black line delineates the combination of these parameters that enter the J96 Coma FP relation.

shape and the shape varies systematically with luminosity (in this context, "strict" homology would imply that all spheroids have the same profile shape). This begs the question of whether this new parameterization of the light profiles has an effect on their location in the FP. To illustrate the effect of using fixed versus varying profile shapes, in Figure 9 we plot the difference between the two structural parameters entering the FP using the best-fit Sérsic profile from the current analysis and the fixed de Vaucouleurs $(n=4)$ fits from T05. The straight line is the bestfit $\log _{10}\left(R_{e}\right)-\mathrm{SBe}$ combination from the J96 local relation for Coma. The E/S0s lie fairly close to the local line, indicating that the FP location of these galaxies is not strongly affected by the shape of the profile. This is due to the fact that, for a given total magnitude, a change in the shape parameter $n$ will result in a different $r_{e}$, which is compensated by a change in SBe in the opposite sense. Thus, regardless of the shape imposed on the fit and the resulting errors on the individual fit parameters, the combination of $r_{e}$ and $\mathrm{SBe}$ entering the FP is a stable quantity (for a similar analysis see Kelson et al. 2000). The relation is not precisely one to one, however, but this would not be expected based on the observed evolution of the FP tilt with redshift (e.g., T05; Jørgensen et al. 2006). On the other hand, there is a significant offset from the $n=4$ fits for the spiral bulges. This is entirely expected as T05 consider single-component $n=4$ fits, whereas we have not only allowed $n$ to vary but also decomposed the profile into bulge and disk components.

Table 4 summarizes the FP parameters derived in the preceding sections for our spectroscopic sample of 147 galaxies.

\section{SPECTROSCOPIC RESULTS}

This section presents our results based on the spectroscopic sample. We first discuss the evolution of the FP in $\S 6.1$ and then interpret it in terms of cosmic evolution of the mass-to-light ratio of bulges and spheroids in $\S 6.2$. In $\S 6.3$ we discuss a number of caveats that should be kept in mind through the discussion of the results and their interpretation in $\S 7$. 
TABLE 4

Fundamental Plane Parameters

\begin{tabular}{|c|c|c|c|c|c|c|c|}
\hline $\begin{array}{l}\text { ID } \\
\text { (1) }\end{array}$ & $\begin{array}{c}n \\
(2)\end{array}$ & $\begin{array}{c}R_{e} \\
(\mathrm{kpc}) \\
(3)\end{array}$ & $\begin{array}{c}\mathrm{SBe} \\
\left(\mathrm{mag}^{\left.\operatorname{arcsec}^{-2}\right)}\right. \\
(4)\end{array}$ & $\begin{array}{c}\sigma_{\mathrm{ap}} \\
\left(\mathrm{km} \mathrm{s}^{-1}\right) \\
(5)\end{array}$ & $\begin{array}{c}B_{\text {rest }} \\
\text { (AB mag) } \\
(6)\end{array}$ & $\begin{array}{c}\log _{10}\left(M / L_{B}\right) \\
\left(M L_{\odot, B}^{-1}\right) \\
(7)\end{array}$ & $\begin{array}{c}\log _{10} M \\
\left(M_{\odot}^{-1}\right) \\
(8)\end{array}$ \\
\hline 198 & $1.4 \pm 0.3$ & $2.31 \pm 0.58$ & $19.40 \pm 0.40$ & $140.0 \pm 8.7$ & -20.78 & $10.91 \pm 0.12$ & $0.38 \pm 0.30$ \\
\hline $280 \ldots \ldots \ldots \ldots \ldots$ & $1.1 \pm 0.3$ & $1.13 \pm 0.39$ & $19.54 \pm 0.60$ & $70.3 \pm 9.2$ & -19.18 & $9.99 \pm 0.19$ & $0.15 \pm 0.43$ \\
\hline $462 \ldots \ldots \ldots \ldots \ldots$ & $1.5 \pm 0.1$ & $1.39 \pm 0.03$ & $19.34 \pm 0.17$ & $230.5 \pm 7.7$ & -19.80 & $11.12 \pm 0.03$ & $1.01 \pm 0.08$ \\
\hline 519 & $1.0 \pm 0.2$ & $2.12 \pm 0.53$ & $20.72 \pm 0.40$ & $75.4 \pm 11.5$ & -19.27 & $10.32 \pm 0.17$ & $0.40 \pm 0.32$ \\
\hline 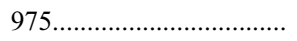 & $1.2 \pm 0.1$ & $1.79 \pm 0.29$ & $20.46 \pm 0.14$ & $81.9 \pm 11.4$ & -18.87 & $10.33 \pm 0.14$ & $0.45 \pm 0.21$ \\
\hline $1050 \ldots \ldots \ldots \ldots \ldots \ldots \ldots \ldots \ldots$ & $1.9 \pm 0.1$ & $2.20 \pm 0.27$ & $19.55 \pm 0.17$ & $240.7 \pm 8.7$ & -20.87 & $11.33 \pm 0.06$ & $0.91 \pm 0.14$ \\
\hline 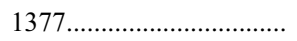 & $1.7 \pm 0.1$ & $2.28 \pm 1.40$ & $20.10 \pm 0.17$ & $170.3 \pm 8.5$ & -20.05 & $11.06 \pm 0.27$ & $0.82 \pm 0.60$ \\
\hline
\end{tabular}

Notes.-This table lists the derived fundamental plane parameters of the spheroidal component of the 147 galaxies in our spectroscopic sample (see $\S 2$ for sample description) as follows. Col. (1): Internal galaxy ID. Col. (2): Sérsic $n$ shape parameter. Col. (3): Effective radius. Col. (4): Average rest-frame $B$-band SB within $R_{e}$. Col. (5): Velocity dispersion as measured in our observed aperture $\left(1^{\prime \prime} \times 0.1185^{\prime \prime}\right)$. Col. (6): Total rest-frame $B$-band AB magnitude of the spheroid. Col. (7): Logarithm of the total $B$-band mass-to-light ratio of the spheroid. Col. (8): Logarithm of the dynamical stellar mass of the spheroid. Table 4 is published in its entirety in the electronic edition of the Astrophysical Journal. A portion is shown here for guidance regarding its form and content.

\subsection{Fundamental Plane Evolution}

With central velocity dispersion measurements in addition to accurate photometric parameters, we now discuss the FP for our sample of intermediate- $z$ spheroids. In Figure 10 we show the location of our spheroids in the face-on projection of the FP according to the J96 local relation for Coma. We divide the sample into five redshift bins [with constant $\Delta(z)$, thus some additional scatter is expected due to the different age ranges in the different bins].

The most notable trend is that with redshift. As redshift increases, bulges and spheroids alike move farther away from the local relation. The mean offset, $\bar{\Delta}_{\mathrm{FP}}$, and the error on the mean are indicated in each panel of Figure 10 and shown as a dotted line. While the trend is not quite systematic with each bin (likely due to a combination of small numbers and the possible overestimation of $\sigma_{0}$ for the lowest $B / T$ systems), the difference between the bins at the extremes is significant. This is consistent with the trend noted previously in T05 for spheroids and can be qualitatively understood in terms of the reduced age of the stellar populations. As cosmic time goes by, stars are on average older, and thus for a given size and velocity dispersion the surface brightness is lower. It is important to keep in mind when comparing with previous studies of early-type galaxies that we have isolated the spheroidal component from the surrounding disk, when present. As a result, galaxies in common with $\mathrm{T} 05$ have generally

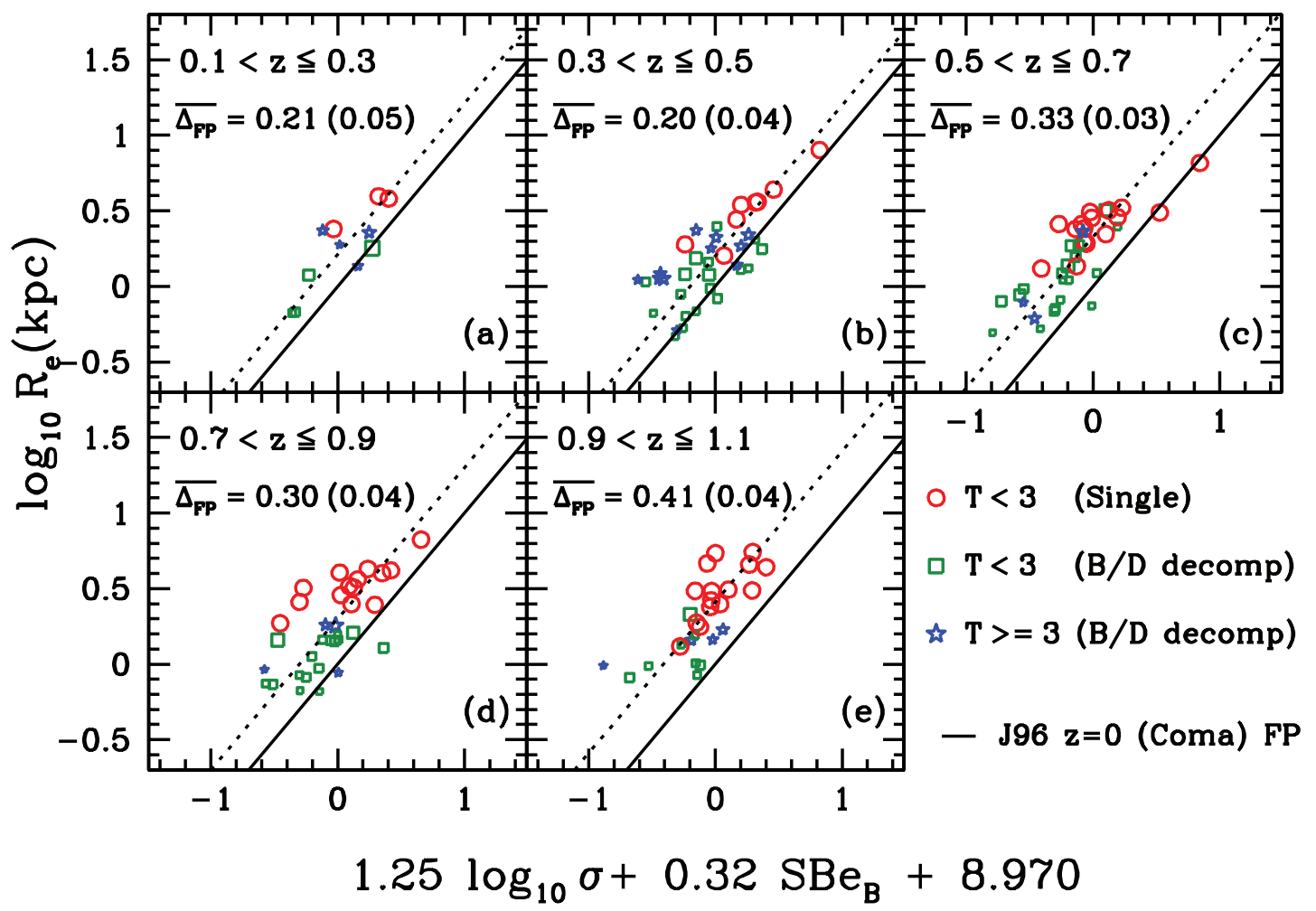

FIG. 10.-FP of our spheroids, divided into five redshift bins, with respect to an edge-on view of the FP set by the local Coma zero point of J96 (solid line in each panel). Point types are indicated, and their size is proportional to the galaxy $B / T$ ratio. Also indicated in each panel is the mean offset from the local FP, $\bar{\Delta}_{\mathrm{FP}}$, and the error on the mean (in brackets). This offset is plotted as the dotted line in each panel. 


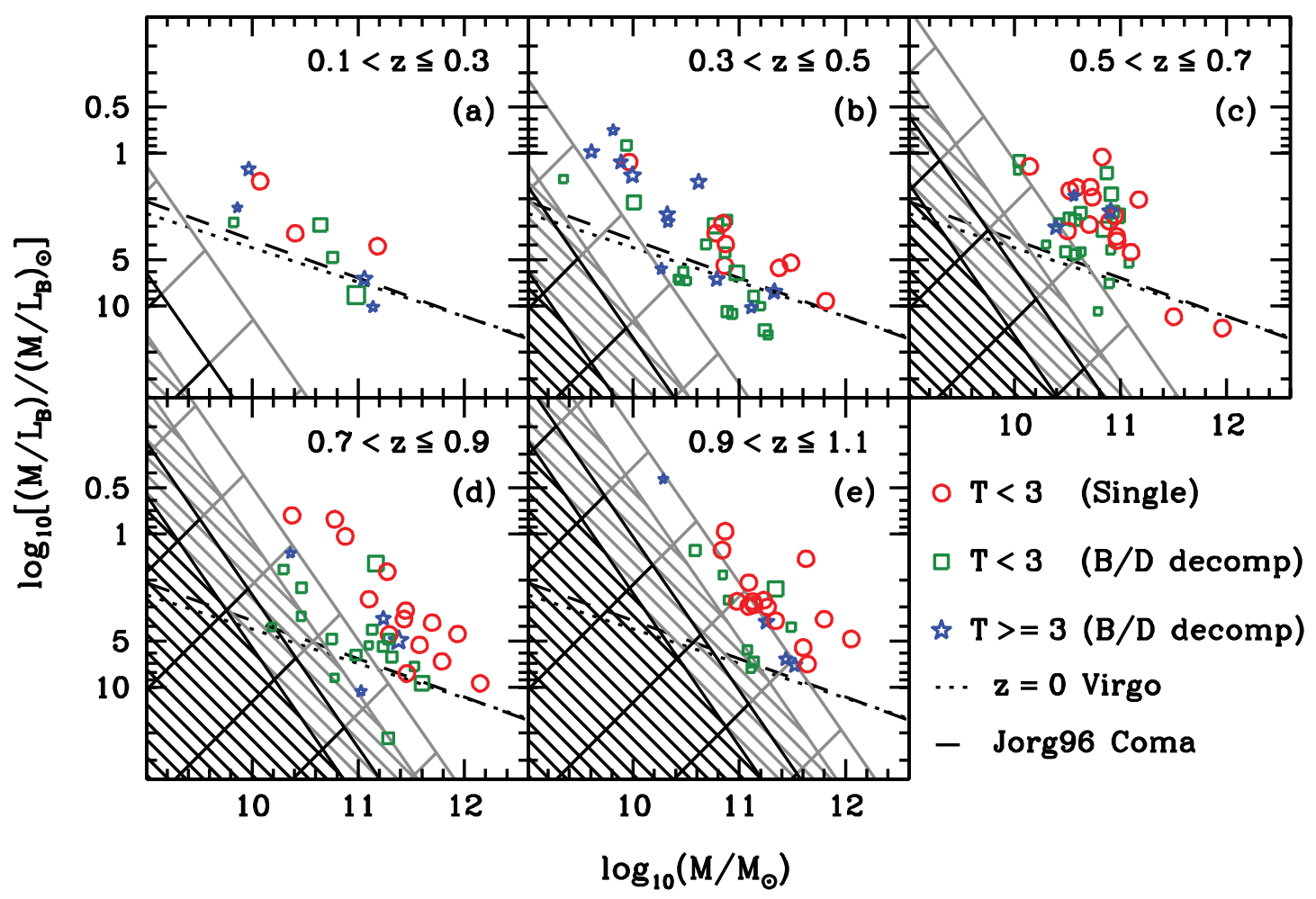

Fig. 11. $-M / L$ vs. $M$ projection of the FP divided into five redshift bins. Panels and symbols are as in Fig. 10. The dotted line is our Virgo local relation (eq. [10]), and the dashed line is that of J96 for Coma. Point size is proportional to $B / T$ ratio. The gray hatched regions correspond roughly to our total galaxy magnitude limit of $z_{\mathrm{AB}}<22.5$. The more densely hatched regions apply to the lower ends of the redshift limits, while the sparsely hatched regions correspond to the upper redshift end. The black hatched regions are the corresponding $z_{\mathrm{AB}}<24.25$ limits on our bulges (i.e., for $B / T=0.2$ ).

moved downward in size (and in luminosity). As expected, the scatter for the spiral bulges is reduced with respect to T05 because we have separated the pressure-supported component from the rotationally supported disk. We note, however, that many of the bulges in the lowest $B / T$ galaxies (point size in Fig. 10 is proportional to $B / T$ ) appear to lie too far to the right of the relation defined by the bigger spheroids. While this effect could be real, we speculate that this is due to the fact that, in the case of twocomponent galaxies, our central velocity dispersions are sensitive to the potential from the total galaxy (bulge/disk/halo) and thus are overestimates of the "bulge-only" dispersions.

Looking at the trends inside each bin, the overall effect seems to imply a continuation in the FP space from the higher mass (E/S0) to the lower mass (bulge) spheroids. However, unlike the trends found in T05, it is not apparent that the FP at higher $z$ is tilted with respect to the local relation, nor that the tilt evolves with redshift.

However, we are still dealing with small numbers per redshift bin, and a confirmation and quantification of this trend (or lack thereof) await a larger sample. The above-mentioned possible overestimation of $\sigma_{0}$ for the lower $B / T$ systems could also be a partial explanation for the apparent lack of a tilt in the higher $z$ FP with respect to the local relation as seen in T05.

\subsection{Mass-to-Light Ratio Evolution}

We now use equation (8) to derive masses for our spheroids and test some of the conjectures above of a correlation between evolutionary state and spheroid mass. In Figure 11 we plot the rest-frame $B$-band mass-to-light ratio $\left(M / L_{B}\right.$; note the inverted scale on the $y$-axis) as a function of mass (both expressed in solar units), separated into the same redshift bins as in Figure 10. The dotted line indicates our Virgo local relation (eq. [10]), and, for comparison, the dashed line shows the J96 relation for Coma that was constructed under the assumption of structural homology. ${ }^{15}$ The shaded areas indicate the selection limits due to our total galaxy magnitude limit of $z_{\mathrm{AB}}<22.5 \mathrm{mag}$. The more densely hatched regions correspond to the lower limit of the redshift bin, while the sparsely hatched regions are for the upper redshift limit. The gray hatches apply to the pure spheroidal galaxies, and the black hatches are the corresponding $z_{\mathrm{AB}}<24.25$ limit for the two-component galaxy sample (i.e., according to our $B / T>0.2$ limit). This selection limit imposes some restrictions on our interpretation of evolution, particularly at the highest redshift and low-mass ends.

Examination of Figure 11 reveals a few interesting trends. Most notably, the observed slope of our relation is somewhat steeper than the local relation slope, even in the lower two redshift bins where selection effects are not important. A linear fit to the (admittedly few) points in the lowest two redshift bins, taking into account errors in both variables, gives slopes of 0.4 and 0.5 , respectively (compared to 0.225 for the local relation). At higher redshift, selection effects become increasingly important and could be responsible for part or all of the observed steepening in the relation (see discussion in T05). Detailed simulations would be needed to quantify whether the apparent change of the tilt can be explained by a combination of increased scatter plus luminosity limits, or whether mass-dependent evolution is indeed present. Given the present uncertainties on the local relation of the spheroidal component and the relatively small size of the present sample, such a detailed quantitative analysis would be premature.

At first glance, it appears that the bulges follow a similar relation to that defined by the pure spheroidal galaxies. In particular,

\footnotetext{
15 These two relations are remarkably consistent with each other and would argue against structural nonhomology as a significant contributer to the FP tilt. Again, however, a confirmation of this awaits a larger sample.
} 


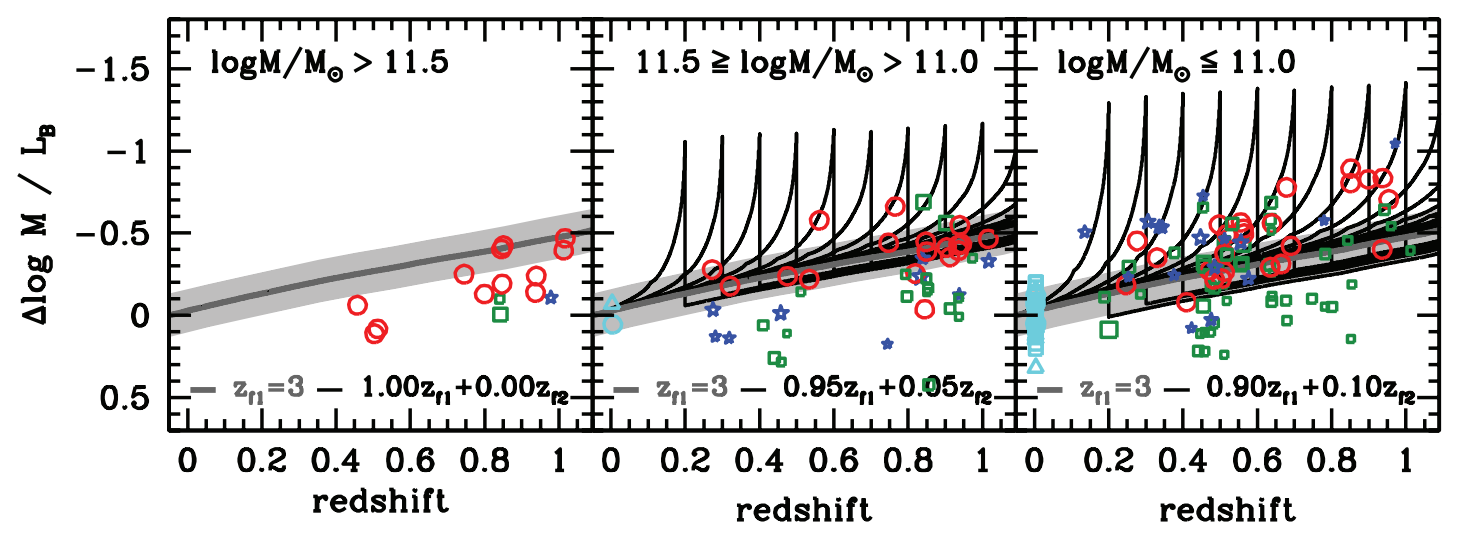

FIg. 12.-Offset of $B$-band $M / L$ ratio, $\Delta \log _{10}\left(M / L_{B}\right)$ (eq. [11]), vs. redshift and divided into three dynamical mass bins. Point size is proportional to $B / T$ ratio. The dark gray line indicates the evolutionary trend for a system that formed in a single burst at $z_{f}=3$ and evolves passively (from the stellar population models of BC03). The light gray band indicates the effect of the error in our local intercept. It is based on the 0.12 dex rms deviation of the local data from the mean relation. Based on the distribution of the most massive spheroids in our intermediate-z sample (left), we note the likelihood of an additional error in the zero point due to calibration errors, both absolute (photometric systems) and from the SDSS $g$ to Johnson $B$ transformation (see the Appendix). Black lines illustrate the effects of secondary burst of SF (5\% or $10 \%$ by mass) at $z_{f 2}=0.1,0.2, \ldots$ added to the initial burst. The light blue points at $z \sim 0$ are the local Virgo galaxies (see the Appendix).

there is no evidence of an offset to lower $M / L$ ratios (due to increased $B$-band luminosity), which may be expected if the bulges are undergoing a more continuous mode of SF, as is expected in the secular formation scenario (e.g., Kormendy \& Kennicutt 2004). If anything, a closer look reveals that some bulges are offset to higher $M / L$. As mentioned above, however, we believe that this could be due to the fact that our central velocity dispersions are sensitive to the total galaxy potential, but our derived masses are based on a formulation for the total mass of a single Sérsic profile. We may, therefore, be overestimating the mass of the spheroid for multiple-component systems. The effect does appear to be stronger for the lower $B / T$ bulges, thus strengthening the support for this hypothesis. Furthermore, selection effects in the higher redshift bins would prevent us from seeing pure spheroids with $M / L$ values as low as some of the two-component systems.

Also evident in Figure 11 is that the deviation from the local relation increases as the spheroid mass decreases. While selection effects prevent us from observing lower mass spheroids with high $M / L$ values for the higher $z$ bins, particularly for the pure spheroids, the fact that we do see strong deviators at the lowmass end but none at the high-mass end (where their observation would not be prevented by selection limits) is still a significant observation. We can represent this offset directly using equation (11). This is shown in Figure 12, which plots $\Delta \log _{10}\left(M / L_{B}\right)$ as a function of redshift and divided into three spheroid mass bins. This representation can be easily interpreted in terms of SFHs in an analogous procedure to that presented in T05 (their Fig. 21). Overplotted on Figure 12 are $M / L$ evolution models from $\mathrm{BC} 03$. The gray lines represent a single burst of SF at $z_{f}=3$ that evolves passively. Based on the distribution of the most massive spheroids, it appears that there is likely a problem with our local relation. This could be in part due to a zero-point calibration error between our data and the local Virgo sample, but it is more likely a manifestation of the fact that our local relation is poorly constrained at the high-mass end; there are no local galaxies in our most massive bin and only two in the intermediate-mass bin (see the Appendix). With the above caveat in mind, the most massive spheroids (including E/S0s and bulges) in the $\log _{10}\left(M / M_{\odot}\right)>$ 11.5 bin are well described by this old population formed in a single burst, based on the relative evolutionary trend with redshift.

In the lower mass bins, however, many galaxies deviate significantly from this relation. These deviations can be explained in the context of more recent spheroid building in the form of subsequent bursts of SF on top of an underlying old population, where the old population dominates the total stellar mass. The black lines in the two lower mass bins represent the $M / L$ evolution for such models. For the intermediate-mass range $[11.5 \leq$ $\left.\log _{10}\left(M / M_{\odot}\right)<11.0\right]$, the data are consistent with a more recent burst of SF that represents $\sim 5 \%$ of the total stellar mass, and in the lowest mass bin, recent bursts involving $\sim 10 \%$ of the total mass are required. Due to the short timescales of the initial $M / L$ decline after a burst of SF, these mass fractions are likely underestimates. These results echo those found in T05 for E/S0s. We emphasize that these models are meant to represent the simplest interpretation of the data. Other SFHs could certainly be constructed to fit the data, but given that these data alone cannot discriminate between the multitude of potential SFHs, the greatest insight is obtained in considering the least complicated case.

Of particular note here is whether the distribution of bulges in the representation shown in Figure 12 is similar to that of the pure spheroidal galaxies. At face value, the distributions in fact appear somewhat different. Indeed, a K-S test comparing the distributions of the offset from the $z_{f}=3$ model relation between the single- and two-component systems confirms that the two populations are drawn from different distributions. However, the difference is driven by the many low- $B / T$ systems that we believe are subject to overestimated masses. If we consider only systems with $B / T>0.4$, the K-S test probability is 0.06 , and for $B / T>0.5$ it is 0.4 , i.e., no statistically significant difference between the two distributions. It is also important to note that it is the upward vertical extent in this figure that implies recent SF activity. In this regard, both samples show the same trend such that higher mass fractions of recent activity are required to explain the vertical distribution of the lower mass systems. Thus, we conclude that for host systems with $B / T>0.2$, all low-mass spheroids, whether isolated or embedded in a disk, must have more recent stellar mass growth, so the recent observations of galaxy "downsizing" in spheroidal galaxies, both empirically (e.g., Bundy et al. 2005; T05; di Serego Alighieri et al. 2005; van der Wel et al. 2005) and in cosmological $N$-body+semianalytical simulations (De Lucia et al. 2006), extend to the bulges of spiral galaxies.

\subsection{Caveats}

Before proceeding to a comparison of our results with other studies in the literature and to a physical interpretation of our spectroscopic and photometric results in the next section, it is 
important to list a number of caveats that should be kept in mind throughout the discussion:

1. Most local FP comparisons are not measured in quite the same way as our distant sample. For example, $r_{e}$ is typically not computed from Sérsic fits, nor are two-component fits generally applied to lenticular galaxies. This may introduce some systematic bias in our determination of the evolution of the mass-tolight ratio. For example, if the slope is steeper locally than we estimate, the deviations in Figure 12 will be tempered, and vice versa. We partially addressed this issue by compiling the FP of Virgo galaxies based on Sérsic fits (F06). However, the Virgo sample is small (smaller in number than the intermediate-redshift sample!), and lenticular galaxies are not decomposed into their spheroidal and disk constituents. Our ongoing Palomar program will hopefully provide us with a more suitable local comparison sample in the near future.

2. Our $\sigma_{0}$ (or $\sigma_{r_{e} / 8}$ ) measurement is going to be sensitive to the total galaxy potential (bulge+disk + halo), so the bulge mass may be systematically overestimated the less prominent the bulge becomes. This could help explain the high $M / L$ ratios measured for our bulges, which appear to be more pronounced for the low- $B / T$ systems, as expected. Although this is beyond the scope of the present observational work, multicomponent dynamical models are needed to determine a more accurate mapping between measured $\sigma_{0}$, effective radius, and bulge stellar mass for low $-B / T$ systems.

3. Significant amounts of dust could also bias high our $M / L$ ratios. If present, one would expect it to be more of an issue the later the Hubble type, and therefore dust could also explain in part the high $M / L$ ratios found for low- $B / T$ systems. A simple way to check for the presence of dust is to study evolutionary trends as a function of inclination. Unfortunately, the present sample is too small to be divided up in bins of the relevant controlling parameters, Hubble type, redshift, inclination, and mass.

4. The main limiting factor of our study is sample size. While collecting data for hundreds of spheroids has been a major effort and represents significant progress with respect to earlier work, in order to detect conclusive trends and address the caveats listed in this section, larger samples are needed at each redshift bin, covering wider (and overlapping across bins) ranges in mass (and luminosity) and Hubble type. It would also be interesting to extend the samples to smaller bulges to verify how far down the mass function the downsizing trend extends.

\section{DISCUSSION}

Having discussed our photometric and spectroscopic results separately in $\S \S 4$ and 5, we are now in a position to combine the inferences from the two diagnostics, compare them with previous studies in the literature, and discuss the physical interpretation.

Colors and mass-to-light ratios provide a consistent picture. The stellar populations of the most massive bulges are homogeneously old (formed at $z>2-3$ ), while an increasingly larger fraction of younger stars (formed below $z \sim 1$ ) is required as one moves down the mass function. This "downsizing" trend is consistent within the errors with that observed for the pure spheroidals and for the spheroidal component of lenticular galaxies. As discussed earlier, this finding allows us to reconcile the results of previous studies of intermediate-redshift bulges based on WFPC2 data (EAD01 and K05).

The results of our study are also consistent with recent studies of the "fossil record" from local samples. Recently, based on the analysis of absorption features, Thomas \& Davies (2006) found that, at a given velocity dispersion, local bulges are indistinguishable from elliptical galaxies in terms of their correlations with age and alpha-element enhancement ratios (as a proxy for SF timescales). Their sample is similarly limited to large $B / T$ spirals.

What does this all imply about bulge formation? It is important to emphasize that different scenarios could lead to the same SFH. For example, for the most massive spheroids analyzed in T05, one cannot distinguish based on stellar populations alone whether the mass assembly happened at high redshift, perhaps concurrent with the major episode of SF, or whether they were assembled at a later time via dry mergers (e.g., van Dokkum 2005; Bell et al. 2006). Independent data, such as those on the evolution of the stellar and dynamical mass functions (e.g., Bundy et al. 2007), are needed to break the degeneracy and conclude that the assembly happened at high redshift as well. For the less massive spheroids, a larger amount of recent SF is required, but understanding whether this happened in situ or via the accretion of gas-rich satellites of mass of order a few times $10^{9} M_{\odot}$ (e.g., T05) again requires external information. The study by Bundy et al. (2007) points toward minor mergers or internal/secular processes as being important alongside major mergers. As far as bulges are concerned then, the similarity in SF need not necessarily reflect a similarity in mechanisms/timing of assembly. At the high-mass end, the homogeneously old stellar populations of bulges seem hard to reconcile with recent formation from existing disk stars or gas. Therefore, it appears that we can safely conclude that massive bulges have been in place well before the redshift range probed by this study, all but ruling out significant secular evolution (see also Thomas \& Davies 2006). Hence, the similarity with spheroids for the most massive bulges appears to be both in the SFH and in the mass assembly history.

At lower masses, the secular process may become more important and it is difficult to disentangle recent in situ SF, mass transfer from the disk to the bulge, and merging with satellites. External evidence suggests that at this redshift and mass scale, bulges are growing significantly via mergers (Woo et al. 2006; Treu et al. 2007), although this may not be the only evolutionary mechanism. If bulges at masses below $10^{11} M_{\odot}$ grow by mergers, then the right panel of Figure 12 must be interpreted with caution: galaxies of a given mass at one redshift are not the progenitors of objects with the same mass at a lower redshift. Perhaps a way to identify the spheroid progenitors is to study bulges at fixed central black hole mass, assuming that growth by accretion is mostly negligible. This is difficult to do in practice and is limited at intermediate redshifts to samples of spheroids hosting active nuclei. However, albeit with large uncertainties, such an exercise (Treu et al. 2007) suggests that spheroids of a few times $10^{10} M_{\odot}$ may have grown in stellar mass by $0.20 \pm 0.14 \mathrm{dex}$ in the past four billion years, while keeping the stellar mass-tolight ratio approximately constant as a result of adding young stars to an aging resident population. The uncertainties are currently too large and the samples too small to perform a quantitative test of this scenario, but it is clear that the evolution of spheroids in this mass range is not purely passive (see also Hopkins et al. 2006) and that complementary information from stellar population studies, spheroid demographics, and scaling relations between host galaxy and central black hole are needed to disentangle SF, assembly history, and black hole growth and feedback. The line of studies presented in this work is crucial to accurately pinpoint the $\mathrm{SFH}$ and could in future provide additional independent information if extended to include tracers of chemical enrichment.

\section{SUMMARY}

We have presented a comprehensive study of field galactic bulges, and of a comparison sample of lenticular and elliptical galaxies, in the redshift range $z=0.1-1.2$. The galaxies are selected 
from GOODS based on visual classification and luminosity $\left(z_{\mathrm{AB}}<\right.$ 22.5). We measure accurate colors and structural parameters for 193 galaxies, fitting two components (bulge and disk) for 137 galaxies and a single Sérsic profile for the remaining 56, classified as pure spheroids. Stellar velocity dispersions are measured from deep spectroscopic observations using DEIMOS on the Keck II telescope. By combining new observations with the measurements derived in T05, we compile a spectroscopic subsample of 147 galaxies suitable for FP analysis, i.e., reliable stellar velocity dispersion and bulge-to-total luminosity ratio greater than $20 \%$, to minimize disk contamination. The spectroscopic subsample includes 56 one-component galaxies (pure spheroids) and 91 twocomponent (bulge+disk) galaxies.

We use the two complementary diagnostics, colors and massto-light ratios as determined from the evolution of the FP, to derive the SFH of bulges and compare it to that of the spheroidal component of lenticular galaxies and of elliptical galaxies. Although the uncertainties are larger for bulges than for pure spheroids ( $\S 6.3$ ), and more data both at intermediate redshift and in the local universe are needed to confirm the trends, the two diagnostics give consistent results that can be summarized as follows:

1. The stellar populations of the more massive bulges $(M>$ $10^{11} M_{\odot}$ ) are homogeneously old, consistent with a single major burst of SF at redshift $\sim 2-3$ or higher and only minor episodes of SF below $z \sim 1$ ( $\sim 5 \%$ in mass $)$.

2. The colors and mass-to-light ratios of smaller bulges $(M<$ $\left.10^{11} M_{\odot}\right)$ span a wider range, consistent with an increasing fraction of younger stars going to smaller masses ( $\sim 10 \%$ in mass).

Although a larger sample is desirable, it appears that the assembly history of bulges and the isolated spheroidal components of lenticular galaxies follow a broadly similar trend to that of pure elliptical galaxies of comparable mass. While differences in their distributions exist in the current analysis, we believe that these are driven by systematic uncertainties in the spheroid mass measurement due to increasing influence of disk and halo mass in the central regions of the galaxy. Future work is planned to help resolve this complicated issue.

Our detection of a mass-dependent SFH allows us to reconcile the findings of two earlier studies of intermediate-redshift bulges based on WFPC2 data (EAD01 and K05). The more massive bulges are as old and red as massive spheroids, but smaller bulges have quite diverse SFHs, with significant SF below $z \sim 1$.
The similarity between the mass assembly history of massive bulges and that of spheroids appears quite naturally explained in a scenario where the former are assembled at high redshift, rather than recently via secular processes within spiral galaxies. At the lower mass end of our sample $\left(M \sim 10^{10} M_{\odot}\right)$, the evolution of bulges below $z \sim 1$ becomes much more diverse, requiring perhaps a combination of merging and secular processes as observed for spheroids of similar mass (Bundy et al. 2007).

This downsizing (e.g., Cowie et al. 1996; Juneau et al. 2005; Bundy et al. 2005; De Lucia et al. 2006; Renzini 2007) picture for bulges extends previous findings based on the integrated properties of galaxies. In addition, this picture is qualitatively consistent with recent results on the coevolution of black holes and spheroids (Treu et al. 2004, 2007; Walter et al. 2004; Woo et al. 2007; Peng 2007), which suggests that bulges in the mass range considered here may have completed their growth after that of the central black hole.

The authors would like to extend their appreciation to Jason Rhodes for running the Tiny Tim PSF simulations and to Stéphane Courteau and Michael McDonald for sharing their XVISTA routines. Thanks also to Ignacio Trujillo, David Koo, Mark Dickinson, and Bob Abraham for useful discussions. We also wish to thank the anonymous referee for a careful review and providing constructive comments that greatly improved the presentation and content of the paper. L. A. M. acknowledges financial support from the National Science and Engineering Council of Canada. T. T. acknowledges support from the NSF through CAREER award NSF-0642621, by the Sloan Foundation through a Sloan Research Fellowship, and by the Packard Foundation through a Packard Fellowship. Some of the data presented herein were obtained at the W. M. Keck Observatory, which is operated as a scientific partnership among the California Institute of Technology, the University of California, and the National Aeronautics and Space Administration. The Observatory was made possible by the generous financial support of the W. M. Keck Foundation. This work is partly based on archival data from the Hubble Space Telescope, obtained from the data archive at the Space Telescope Institute, which is operated by the Association of Universities for Research in Astronomy, Inc., for NASA under contract NAS5-26555.

\section{APPENDIX}

\section{DERIVATION OF LOCAL VIRGO FUNDAMENTAL PLANE}

A crucial aspect for the above evolutionary study is a local zero point against which we can compare our higher $z$ galaxies. In order to obtain a meaningful comparison, strict homogeneity in the data and analysis techniques is required. There do exist a few local FP standards in the literature. One of the most commonly used is the FP of J96 based on Coma Cluster early-type galaxies. This FP was derived under the assumption of structural homology, fitting all galaxies with a single de Vaucouleurs profile. In this case, the virial coefficient is a constant for all galaxies and the FP maps directly into an $M / L$ ratio (e.g., T05). However, in the case of varying $n$, the profile shape does affect the $M / L$ ratio through a variation in the $\sigma(r)$ profile. In the current study we have relaxed the assumption of structural homology and fitted all spheroids with the more general Sérsic profile with varying $n$. The J96 relation is thus not appropriate as a local comparison relation here.

We seek a local sample for which galaxy profiles are modeled with the Sérsic profile and comprise a homogeneous database of velocity dispersion measurements. As far as we are aware, the only analysis to date that provides a local $M / L$ versus $M$ relation, taking into account varying profile shapes, is that of T04. Their FP sample includes 45 cluster ellipticals (from Virgo, Fornax, and Coma). The photometric parameters were obtained from the literature and the velocity dispersions from the Hypercat ${ }^{16}$ database. The sample in that analysis is quite inhomogeneous and could not make use of more recent accurate distance measurements to Virgo Cluster galaxies, thus rendering it an unsatisfying zero point from which to compare our distant sample.

\footnotetext{
16 See http://leda.univ-lyon1.fr.
} 

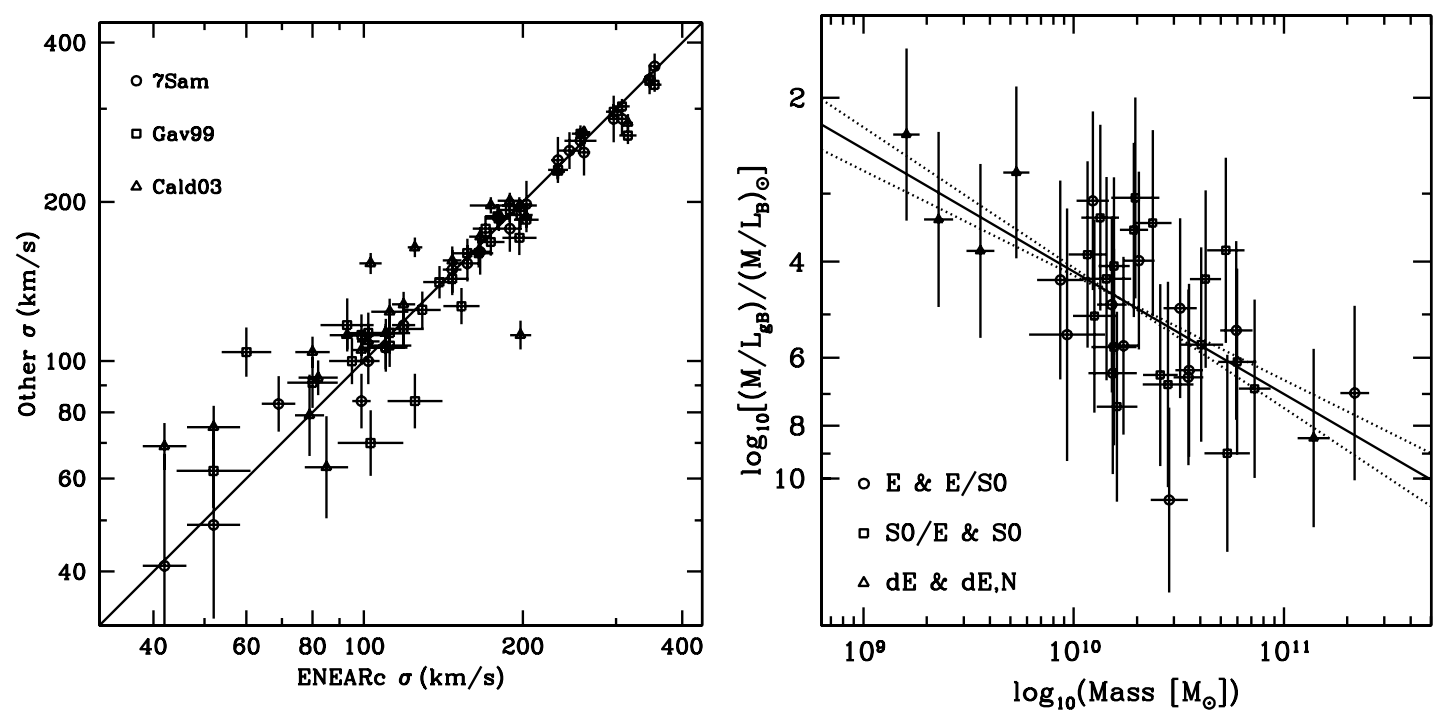

FIG. 13. - Left: Comparison of literature velocity dispersion measurements used in our compilation of Virgo early-type galaxies. The solid line is the one-to-one relation. Right: Virgo FP projected onto the $M / L$ vs. $M$ plane using equation (8). The solid line is the orthogonal fit to the data, and the dotted lines represent the fit errors.

Recently, however, the ACSVCS of Côté et al. (2004) has obtained ACS images in the F475W and F850LP bandpasses of 100 earlytype galaxies in the Virgo Cluster. F06 present a detailed structural analysis of these galaxies modeling their SB profiles with Sérsic models. Furthermore, Mei et al. (2007) present precise distance measurements to 84 of these galaxies based on surface brightness fluctuations (SBFs). Accurate distance measurements are crucial for conversion to physical parameters for FP analyses. Given the significant line-of-sight depth and subclustering of the Virgo Cluster, we restrict ourselves to these 84 galaxies with accurate SBF distance measurements.

While the F475W $(g)$ band is close to $B$, they are not identical and a calibration between the two is required. F06 tabulate the total $B$ magnitudes from the VCC survey of Binggeli et al. (1985) and in their Figure 115 plot these $B$ magnitudes against their total $g$, computed by integrating the Sérsic fit parameters to infinity. The fainter galaxies closely follow the one-to-one relation. Moving to brighter systems, there is a systematic trend to brighter $g$ magnitudes. F06 associate this with observationally established color-magnitude trends (becoming redder with higher luminosity). However, at the brightest end, the "core" galaxies, whose light profiles within the central few hundred parsecs fall below an inward extrapolation of the "outer" Sérsic fit (for which the central points were omitted), deviate strongly in the sense that the fit-derived magnitudes are significantly brighter than those derived "from the data." This deviation seems too strong to be attributed to stellar population effects alone.

As a consistency check, we compared the $g-B$ versus $g-z$ colors with the stellar population models of BC03. The data span a reasonable range in $g-z$ in the context of stellar populations, but the $g-B$ colors scatter significantly off the model grids, indicating a calibration issue between the ACSVCS and Binggeli et al. (1985) photometry. Most galaxies scatter to the blue in $B-g$, but all of the "core" galaxies scatter to the red (again indicating an overestimated $g$ magnitude). This color plane is fairly degenerate in metallicity; thus, a simple linear relation can be used to convert from the observed $g$ band to $B$ band. We adopted the conversion $B=g+$ $[0.25(g-z)-0.075]$ and refer to this as the " $g B$ " magnitude.

An independent check on the ACSVCS photometric analysis was made by comparing their results to 23 galaxies in common with the study of Gavazzi et al. (2005), who present $B$-band Sérsic modeling of 226 giant and dwarf elliptical Virgo Cluster galaxies located in the northeast quadrant. The agreement between structural parameters is quite good for all noncore galaxies. In addition, at observed magnitudes fainter than $B \sim 11$, our derived $g B$ magnitudes agree very well with the Gavazzi et al. (2005) total $B$-band magnitudes, which were derived "from the data" rather than computed from the fit parameters. The core galaxies show significant discrepancies in total magnitude and the photometric parameters.

A number of studies have pointed out unique properties of core elliptical galaxies when compared with the dE/E sequences. They define a different and quite distinct photometric plane than dE/Es (e.g., Graham \& Guzmán 2003; Gavazzi et al. 2005; F06). Many argue that the observed cores arise from the partial evacuation of the nuclear region by coalescing black holes (e.g., Merritt 2006). Core ellipticals are also relatively rare (i.e., they are the exception rather than the rule), even in a cluster environment, and are presumably even less common in the field. As such, we likely do not have any core ellipticals in our field sample (although we cannot tell for sure due to insufficient resolution for these high- $z$ galaxies). All of the above argues against including these outliers in our local sample; thus, we also exclude all galaxies identified as "core Sérsic" in F06. This leaves us with 74 Virgo early-type galaxies with accurate photometric decompositions and distance measurements.

We then searched the literature and HyperLeda database for velocity dispersion measurements for this sample to construct a local FP. Given the strict requirement of uniformity, we were scrupulous in culling the velocity dispersion measurement among different studies. In the end, we included $\sigma$-values from five different samples: Bernardi et al. (2002; ENEARc), Faber et al. (1989; 7Sam), Gavazzi et al. (1999; Gav99), Caldwell et al. (2003; Cald03), and Geha et al. (2003). Velocity dispersions were selected using the following hierarchy: ENEARc if $\sigma>75 \mathrm{~km} \mathrm{~s}^{-1}$, 7Sam if $\sigma>75 \mathrm{~km} \mathrm{~s}^{-1}$, Gav99 if $\sigma>75 \mathrm{~km} \mathrm{~s}^{-1}$, Cald03 if $\sigma<100 \mathrm{~km} \mathrm{~s}^{-1}$. The left panel of Figure 13 compares the literature values from the above studies for common galaxies. The agreement is quite good at large $\sigma$ but starts to break down at $\sigma \lesssim 100 \mathrm{~km} \mathrm{~s}^{-1}$. 
Finally, we included all velocity dispersion measurements for dEs in common with the study of Geha et al. (2003) (without restrictions). We are thus left with a final Virgo FP relation including $36 \mathrm{E} / \mathrm{S} 0 / \mathrm{dE}$ galaxies.

Corrections to an aperture of $r_{e} / 8$ were made as above (see $\S 5.3$ ) assuming a $2^{\prime \prime}$ aperture for all samples except that of Geha et al. (2003), for which we adopt a $1^{\prime \prime}$ aperture.

With this final sample of 36 noncored early-type Virgo galaxies we construct the $M / L_{B}$ versus $M$ projection of the FP using equation (8). An orthogonal fit to the data using the procedure of Akritas \& Bershady (1996) provides the relation

$$
\log _{10}\left(M / L_{B}\right)_{0}=(0.225 \pm 0.033) \log _{10}(M)-(1.63 \pm 0.34) .
$$

We use this relation as the local comparison for our higher $z$ sample in $\S 6.2$. We note, however, that our local relation remains poorly constrained. This is due to the small number of galaxies surviving the pruning process required to achieve a reliably homogeneous sample and, more importantly, due to the dearth of high-mass objects remaining, restricting the dynamic range of the fit. We will address this issue in forthcoming publications with the aid of an enlarged local sample.

Aguerri, J. A. L., Balcells, M., \& Peletier, R. F. 2001, A\&A, 367, 428 Akritas, M. G., \& Bershady, M. A. 1996, ApJ, 470, 706

Andredakis, Y. C., Peletier, R. F., \& Balcells, M. 1995, MNRAS, 275, 874 Baugh, C. M., Cole, S., Frenk, C. S., \& Lacey, C. G. 1998, ApJ, 498, 504 Bell, E. F., et al. 2006, ApJ, 640, 241

Bernardi, M., Alonso, M. V., da Costa, L. N., Willmer, C. N. A., Wegner, G.,

Pellegrini, P. S., Rité, C., \& Maia, M. A. G. 2002, AJ, 123, 2990

Bertin, E., \& Arnouts, S. 1996, A\&AS, 117, 393

Bertin, G., Ciotti, L., \& Del Principe, M. 2002, A\&A, 386, 149

Binggeli, B., Sandage, A., \& Tammann, G. A. 1985, AJ, 90, 1681

Blanton, M. R., \& Roweis, S. 2007, AJ, 133, 734

Bolton, A. S., Burles, S., Treu, T., Koopmans, L. V. E., \& Moustakas, L. A. 2007, ApJ, 665, L105

Bower, R. G., Lucey, J. R., \& Ellis, R. S. 1992, MNRAS, 254, 601

Bruzual, A. G., \& Charlot, S. 2003, MNRAS, 344, 1000 (BC03)

Bundy, K., Ellis, R. S., \& Conselice, C. J. 2005, ApJ, 625, 621

Bundy, K., Treu, T., \& Ellis, R. S. 2007, ApJ, 665, L5

Caldwell, N., Rose, J. A., \& Concannon, K. D. 2003, AJ, 125, 2891

Caon, N., Capaccioli, M., \& D’Onofrio, M. 1993, MNRAS, 265, 1013

Cappellari, M., et al. 2006, MNRAS, 366, 1126

Charlot, S., \& Fall, S. M. 2000, ApJ, 539, 718

Ciotti, L., Lanzoni, B., \& Renzini, A. 1996, MNRAS, 282, 1

Coelho, P., Barbuy, B., Meléndez, J., Schiavon, R. P., \& Castilho, B. V. 2005, A\&A, 443, 735

Combes, F. 2007, in IAU Symp. 235, Galaxy Evolution across the Hubble

Time, ed. F. Combes \& J. Palous (Cambridge: Cambridge Univ. Press), 19

Côté, P., et al. 2004, ApJS, 153, 223

Courteau, S., de Jong, R. S., \& Broeils, A. H. 1996, ApJ, 457, L73

Cowie, L. L., Songaila, A., Hu, E. M., \& Cohen, J. G. 1996, AJ, 112, 839

Davis, M., et al. 2003, Proc. SPIE, 4834, 161

Debattista, V. P., Mayer, L., Carollo, C. M., Moore, B., Wadsley, J., \& Quinn, T. 2006, ApJ, 645, 209

de Jong, R. S. 1996, A\&AS, 118, 557

De Lucia, G., Springel, V., White, S. D. M., Croton, D., \& Kauffmann, G. 2006, MNRAS, 366, 499

di Serego Alighieri, S., et al. 2005, A\&A, 442, 125

Djorgovski, S., \& Davis, M. 1987, ApJ, 313, 59

Dressler, A., Lynden-Bell, D., Burstein, D., Davies, R. L., Faber, S. M., Terlevich, R., \& Wegner, G. 1987, ApJ, 313, 42

Eggen, O. J., Lynden-Bell, D., \& Sandage, A. R. 1962, ApJ, 136, 748

Eliche-Moral, M. C., Balcells, M., Aguerri, J. A. L., \& González-García, A. C. 2006, A\&A, 457, 91

Ellis, R. S., Abraham, R. G., \& Dickinson, M. 2001, ApJ, 551, 111 (EAD01)

Elmegreen, B. G., Elmegreen, D. M., Vollbach, D. R., Foster, E. R., \& Ferguson, T. E. 2005, ApJ, 634, 101

Faber, S. M., Wegner, G., Burstein, D., Davies, R. L., Dressler, A., Lynden-Bell, D., \& Terlevich, R. J. 1989, ApJS, 69, 763

Faber, S. M., et al. 2003, Proc. SPIE, 4841, 1657

Ferrarese, L., et al. 2006, ApJS, 164, 334 (F06)

Freeman, K. C. 1970, ApJ, 160, 811

Gavazzi, G., Boselli, A., Scodeggio, M., Pierini, D., \& Belsole, E. 1999, MNRAS, 304, 595

Gavazzi, G., Donati, A., Cucciati, O., Sabatini, S., Boselli, A., Davies, J., \& Zibetti, S. 2005, A\&A, 430, 411

Gebhardt, K., et al. 2000, ApJ, 543, L5 2003, ApJ, 597, 239

Geha, M., Guhathakurta, P., \& van der Marel, R. P. 2003, AJ, 126, 1794

Giavalisco, M., et al. 2004, ApJ, 600, L93

González, J. J. 1993, Ph.D. thesis, Univ. California, Santa Cruz
Graham, A. W. 2001, AJ, 121, 820

Graham, A. W., \& Guzmán, R. 2003, AJ, 125, 2936

Hopkins, P. F., Somerville, R. S., Hernquist, L., Cox, T. J., Robertson, B., \& Li, Y. 2006, ApJ, 652, 864

Jørgensen, I., Chiboucas, K., Flint, K., Bergmann, M., Barr, J., \& Davies, R. 2006, ApJ, 639, L9

Jørgensen, I., Franx, M., \& Kjaergaard, P. 1996, MNRAS, 280, 167 (J96)

Juneau, S., et al. 2005, ApJ, 619, L135

Kauffmann, G., Charlot, S., \& White, S. D. M. 1996, MNRAS, 283, L117

Kelson, D. D., Illingworth, G. D., van Dokkum, P. G., \& Franx, M. 2000, ApJ, 531,137

Koo, D. C., et al. 2005, ApJS, 157, 175 (K05)

Kormendy, J. 1977, ApJ, 217, 406

-. 1985, ApJ, 295, 73

Kormendy, J., \& Kennicutt, R. C., Jr. 2004, ARA\&A, 42, 603

La Barbera, F., Busarello, G., Merluzzi, P., Massarotti, M., \& Capaccioli, M. 2003, ApJ, 595, 127

MacArthur, L. A., Courteau, S., Bell, E., \& Holtzman, J. A. 2004, ApJS, 152, 175

MacArthur, L. A., Courteau, S., \& Holtzman, J. A. 2003, ApJ, 582, 689 (M03)

Magorrian, J., et al. 1998, AJ, 115, 2285

Mei, S., et al. 2007, ApJ, 655, 144

Merritt, D. 2006, Rep. Prog. Phys., 69, 2513

Miralda-Escudé, J., \& Kollmeier, J. A. 2005, ApJ, 619, 30

Nipoti, C., Londrillo, P., \& Ciotti, L. 2006, MNRAS, 370, 681

Oke, J. B. 1974, ApJS, 27, 21

Peng, C. Y. 2007, ApJ, 671, 1098

Prugniel, P., \& Simien, F. 1997, A\&A, 321, 111

Ravikumar, C. D., Barway, S., Kembhavi, A., Mobasher, B., \& Kuriakose, V. C. 2006, A\&A, 446, 827

Renzini, A. 2007, in ASP Conf. Ser. 380, At the Edge of the Universe, ed. J. Afonso et al. (San Francisco: ASP), 309

Rhodes, J. D., et al. 2007, ApJS, 172, 203

Scannapieco, C., \& Tissera, P. B. 2003, MNRAS, 338, 880

Schade, D., Carlberg, R. G., Yee, H. K. C., Lopez-Cruz, O., \& Ellingson, E. 1996, ApJ, 464, L63

Schlegel, D. J., Finkbeiner, D. P., \& Davis, M. 1998, ApJ, 500, 525

Sérsic, J. L. 1969, Atlas de Balaxias Australes (Cordoba: Obs. Astronomico)

Sirianni, M., et al. 1998, Proc. SPIE, 3355, 608

Thomas, D., \& Davies, R. L. 2006, MNRAS, 366, 510

Treu, T., Ellis, R. S., Liao, T. X., \& van Dokkum, P. G. 2005a, ApJ, 622, L5

Treu, T., Malkan, M. A., \& Blandford, R. D. 2004, ApJ, 615, L97

Treu, T., Stiavelli, M., Casertano, S., Møller, P., \& Bertin, G. 2002, ApJ, 564, L13

Treu, T., Stiavelli, M., Møller, P., Casertano, S., \& Bertin, G. 2001, MNRAS, 326,221

Treu, T., Woo, J.-H., Malkan, M. A., \& Blandford, R. D. 2007, ApJ, 667, 117

Treu, T., et al. 2005b, ApJ, 633, 174 (T05)

Trujillo, I., Burkert, A., \& Bell, E. F. 2004, ApJ, 600, L39 (T04)

van der Marel, R. P. 1994, MNRAS, 270, 271

van der Wel, A., Franx, M., van Dokkum, P. G., Rix, H.-W., Illingworth, G. D., \& Rosati, P. 2005, ApJ, 631, 145

van Dokkum, P. G. 2005, AJ, 130, 2647

van Dokkum, P. G., \& Ellis, R. S. 2003, ApJ, 592, L53

Walter, F., Carilli, C., Bertoldi, F., Menten, K., Cox, P., Lo, K. Y., Fan, X., \& Strauss, M. A. 2004, ApJ, 615, L17

Widrow, L. M., \& Dubinski, J. 2005, ApJ, 631, 838

Wirth, G. D., et al. 2004, AJ, 127, 3121

Woo, J.-H., Treu, T., Malkan, M. A., \& Blandford, R. D. 2006, ApJ, 645, 900 Woo, J.-H., Treu, T., Malkan, M. A., Ferry, M. A., \& Misch, T. 2007, ApJ, 661, 60 Wyse, R. F. G., Gilmore, G., \& Franx, M. 1997, ARA\&A, 35, 637 